CERN-PH-EP/2007-030

10 August 2007

\title{
THE HARP TIME PROJECTION CHAMBER: CHARACTERISTICS AND PHYSICS PERFORMANCE
}

\begin{abstract}
The HARP spectrometer that took data at the CERN Proton Synchrotron in 2001 and 2002 had as large-angle detector system a Time Projection Chamber (TPC) surrounded by Resistive Plate Chambers. The design of the TPC, experience with its operation, and its good physics performance are described. The successful recovery from track distortions arising from inhomogeneities of the electric and magnetic fields in the TPC volume is discussed.
\end{abstract}

A. Bolshakova, I. Boyko, G. Chelkov, D. Dedovitch, A. Elagin ${ }^{1}$, M. Gostkin, A. Guskov, Z. Kroumchtein, Yu. Nefedov, K. Nikolaev, A. Zhemchugov

Joint Institute for Nuclear Research, Dubna, Russia

F. Dydak, L. Linssen, J. Wotschack CERN, Geneva, Switzerland

\author{
A. De $\mathrm{Min}^{2}$ \\ Politecnico di Milano and INFN, Sezione di Milano-Bicocca, Italy \\ V. Ammosov, V. Gapienko, V. Koreshev, A. Semak, Yu. Sviridov, V. Zaets \\ Institute for High Energy Physics, Protvino, Russia
}

(To be submitted to Nucl. Instrum. Methods Phys. Res. A)

\footnotetext{
${ }^{1}$ Now at Texas A\&M University, College Station, USA.

${ }^{2}$ On leave of absence at Ecole Polytechnique Fédérale, Lausanne, Switzerland.
} 


\section{Contents}

1 Introduction 3

2 Performance: objectives and results 3

3 TPC construction and operation 3

3.1 Mechanical design . . . . . . . . . . . . . . . . . 4

3.2 Solenoidal magnetic field . . . . . . . . . . . . . . . . . . 8

3.3 Gas parameters ......................... 8

3.4 Readout electronics . . . . . . . . . . . . . . . . . . . . . . 11

4 From raw data to physics parameters 11

4.1 From time samples to cluster coordinates . . . . . . . . . . . . . . . 11

4.2 Pad pulse-height normalization . . . . . . . . . . . . . . 11

4.3 From clusters to tracks . . . . . . . . . . . . . . . . . . . 12

4.4 Helix fit of tracks . . . . . . . . . . . . . . . . . . . . . 12

4.5 Azimuthal coordinate precision . . . . . . . . . . . . . . . 13

$4.6 p_{\mathrm{T}}$ resolution and charge identification . . . . . . . . . . . . 14

4.7 Longitudinal coordinate precision and polar-angle resolution . . . . . . . . 15

$4.8 \mathrm{~d} E / \mathrm{d} x$ resolution $\ldots \ldots \ldots \ldots \ldots \ldots \ldots$

5 Recovery from track distortions $\quad 16$

5.1 Distortions from electronics cross-talk . . . . . . . . . . . . . . . 16

5.2 Distortions from field inhomogeneities . . . . . . . . . . . . 18

5.2 .1 Static distortions . . . . . . . . . . . . . . 20

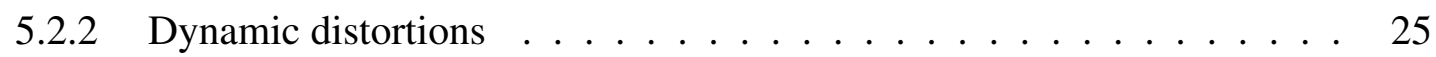

5.2.3 Synopsis of distortions from field inhomogeneities . . . . . . . . . 32

6 Summary of the physics performance 32

6.1 Momentum scale . . . . . . . . . . . . . . . . . . 32

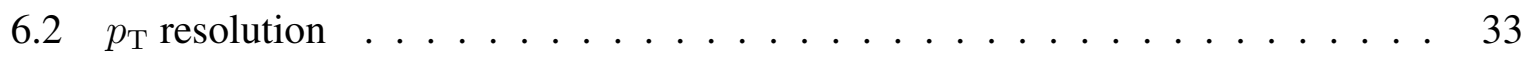

$6.3 \mathrm{~d} E / \mathrm{d} x$ versus momentum . . . . . . . . . . . . . . . . . . 33

6.4 Time of flight versus momentum . . . . . . . . . . . . . . . . . . . . . . . . . . 33

6.5 Pion-electron separation $\ldots \ldots \ldots \ldots$

$\begin{array}{lll}7 & \text { Summary } & 37\end{array}$ 


\section{INTRODUCTION}

The HARP experiment arose from the realization that the differential cross-sections of hadron production in the collisions of low-momentum protons with nuclei were known only within a factor of two to three. Consequently, the HARP spectrometer was designed to carry out a programme of systematic and precise measurements of hadron production by protons and pions with momenta from 1.5 to $15 \mathrm{GeV} / c$. The experiment was performed at the CERN Proton Synchrotron in 2001 and 2002 with a set of targets ranging from hydrogen to lead.

With a view to achieving nearly $4 \pi$ acceptance, the HARP detector combined a large-angle spectrometer with a forward spectrometer. The latter consisted of a dipole magnet and drift chambers for momentum measurement, and a threshold Cherenkov counter, a time-of-flight wall, and an electromagnetic calorimeter for particle identification. The large-angle spectrometer comprised a cylindrical Time Projection Chamber (TPC) and an array of Resistive Plate Chambers (RPCs) around and behind the TPC. The purpose of the TPC was track reconstruction and particle identification by $\mathrm{d} E / \mathrm{d} x$. The purpose of the RPCs was to complement the particle identification by $\mathrm{d} E / \mathrm{d} x$ by time of flight, especially to distinguish between electrons and pions in the momentum range $150-250 \mathrm{MeV} / \mathrm{c}$ where the specific ionization of electrons and pions is too close for their separation.

This paper describes the HARP TPC, the experience with its operation, and its physics performance.

The TPC had to be designed, constructed and commissioned within a brief period of 17 months. Under this time pressure a few per se minor mishaps occurred which, however, had quite some consequences for the quality of the raw data. Part of this paper is dedicated to the - so we hope-interesting discovery of, and the recovery from, these errors.

This paper also sets the record straight with respect to the TPC performance reported in Refs. [1] and [2]. It justifies and details our criticism thereof [3,4].

\section{Performance: OBJectives and Results}

The experiment was carried out in the T9 beam of the CERN Proton Synchrotron that had a maximum momentum of $p=15 \mathrm{GeV} / c$. Thus in the large-angle region, the detector was to handle comfortably transverse momenta up to $p_{\mathrm{T}} \sim 2 \mathrm{GeV} / c$. A, say, $2 \sigma$ separation $(2.3 \%$ misidentification probability) between positive and negative charges at $p_{\mathrm{T}}=2 \mathrm{GeV} / c$ called for a resolution $\sigma\left(1 / p_{\mathrm{T}}\right)$ not worse than about $0.25(\mathrm{GeV} / c)^{-1}$.

Table 1 summarizes the key parameters of the TPC, and compares objectives with the achieved results. In later sections, the table entries will be discussed in detail ${ }^{1)}$.

\section{TPC CONSTRUCTION AND OPERATION}

In HARP's large-angle region, the tracking detector of choice was a cylindrical TPC.

The decision to re-use the solenoidal 'TPC90' magnet [5] of the ALEPH Collaboration constrained the dimensions of the TPC. The magnet had an inner bore of $90 \mathrm{~cm}$. With a view to ensuring a homogeneous magnetic field over a sufficient length, the original magnet was longitudinally extended by $500 \mathrm{~mm}$. The upstream end was closed by a $150 \mathrm{~mm}$ thick iron plate, leaving only a $150 \mathrm{~mm}$ diameter entry hole for the beam, while the rear end of the solenoid was left open in order not to obstruct the entrance into HARP's forward spectrometer. The flux

\footnotetext{
1) A right-handed Cartesian and/or spherical polar coordinate system is employed: looking downstream in the $+z$ direction, the $+x$ coordinate points to the left and the $+y$ coordinate points up; the polar angle $\theta$ is the angle with respect to the $+z$ axis; when looking downstream, the azimuthal angle $\phi$ increases in the clockwise direction, with the $+x$ axis at $\phi=0$.
} 
Table 1: Objectives and achieved results of the key performance parameters of the HARP TPC, originating from statistical or quasi-statistical fluctuations; for details we refer to Sections 4.5 to 4.8.

\begin{tabular}{|l|c|c|}
\hline & Objective & Result \\
\hline \hline$r \cdot \phi$ resolution $[\mu \mathrm{m}]$ & 360 & $600-2400$ \\
\hline from wire angular and wire $E \times B$ effects $[\mu \mathrm{m}]$ & $0-200$ & \\
from transverse diffusion over $1 \mathrm{~m}[\mu \mathrm{m}]$ & 130 & \\
from the pad angular effect $[\mu \mathrm{m}]$ & 190 & \\
from digitization $[\mu \mathrm{m}]$ & 140 & \\
from FADC threshold and electronics noise $[\mu \mathrm{m}]$ & 70 & \\
from ${ }^{83 \mathrm{~m}} \mathrm{Kr}$ calibration and preamplifier non-linearity $[\mu \mathrm{m}]$ & 110 & \\
from uni- and/or bidirectional cross-talk $[\mu \mathrm{m}]$ & & $0-2000$ \\
from $\leq 3$ pads per cluster $[\mu \mathrm{m}]$ & & $110-1300$ \\
\hline \hline $1 / p_{\mathrm{T}}$ resolution $\left[(\mathrm{GeV} / c)^{-1}\right]$ without beam point & $\sim 0.18$ & $0.45-0.50$ \\
$1 / p_{\mathrm{T}}$ resolution $\left[(\mathrm{GeV} / c)^{-1}\right]$ with beam point & $\sim 0.14$ & $0.20-0.25$ \\
\hline \hline$z$ resolution [mm] & 2.6 & $\sim 3.5$ \\
\hline from longitudinal diffusion over $0.5 \mathrm{~m}[\mathrm{~mm}]$ & 0.33 & \\
from digitization and FADC threshold $[\mathrm{mm}]$ & 2.6 & \\
from self cross-talk [mm] & & $\sim 2.3$ \\
\hline \hline$\theta$ resolution for $\theta=60^{\circ}[\mathrm{mrad}]$ & 6.6 & $\sim 9$ \\
\hline \hline $\mathrm{d} E / \mathrm{d} x$ resolution over $300 \mathrm{~mm}[\%]$ & $\sim 16$ & 16 \\
\hline
\end{tabular}

return consisted of 16 iron slabs of $200 \times 200 \mathrm{~mm}^{2}$ running along the full length covered by the coils. For maximum acceptance of secondary particles, the target was located inside the TPC.

The TPC filled most of the inner bore of the magnet, leaving a $25 \mathrm{~mm}$ wide gap between TPC and magnet coils. This gap was used to house two overlapping layers of $2000 \mathrm{~mm}$ long RPCs [6] directly mounted onto the outer field cage of the TPC. Figure 1 shows a photograph of the TPC together with the surrounding RPCs at the moment of insertion into the magnet.

\subsection{Mechanical design}

The layout of the TPC and its position in the solenoidal magnet is shown in Fig. 2. The TPC has an external diameter of $832 \mathrm{~mm}$ and an overall length of $\sim 2000 \mathrm{~mm}$. It consists of two Stesalit cylinders forming the inner and outer field cages, a wire chamber with pad readout, located at the upstream end, and a high-voltage (HV) membrane at $1567 \mathrm{~mm}$ distance from the pad plane. The inner field cage (IFC) extends over about half of the drift volume; its end is closed by a thin aluminized Mylar foil, serving as HV membrane. It encloses the target, the centre of which is located $500 \mathrm{~mm}$ downstream of the pad plane.

An electrical drift field of $111.1 \mathrm{~V} / \mathrm{cm}$ was chosen as operating point since it provides in the chosen gas mixture (see Section 3.3) for maximum electron drift velocity and hence minimal dependence on temperature and pressure changes. It was achieved by a potential of $-17302 \mathrm{~V}$ at the HV membrane at the downstream end of the outer field cage (OFC). This potential was degraded through $10 \mathrm{~mm}$ wide potential strips, connected to a chain of $0.5 \mathrm{M} \Omega$ resistors, over the full length of the drift volume to zero potential at the cathode wire plane. The potential strips were arranged in two layers, shifted longitudinally by $5 \mathrm{~mm}$ with respect to each other. 


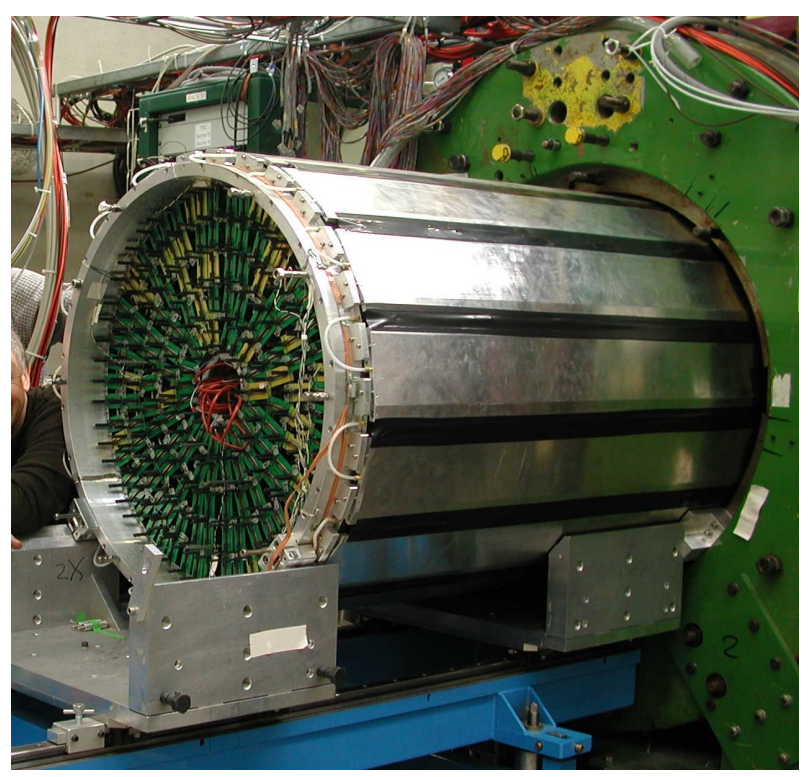

Fig. 1: TPC, still without cabling, surrounded by the RPCs, at the moment of insertion into the magnet.

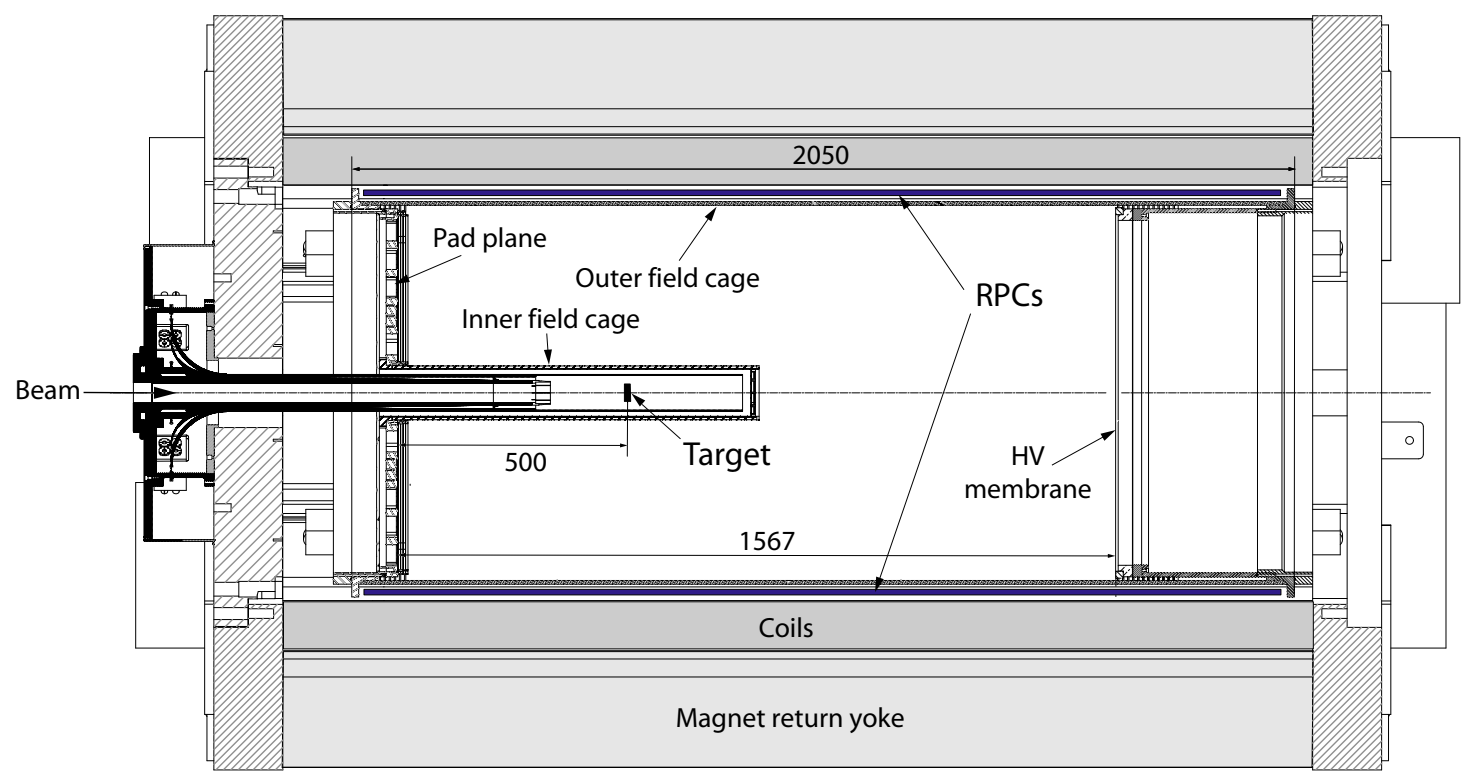

Fig. 2: Cut through TPC and solenoidal magnet; the beam enters from the left side. 
The first layer consisted of copper strips directly on the Stesalit cylinders, the second layer was realized with aluminized Mylar strips supported by thin Stesalit rods glued longitudinally onto the field cage cylinders. The potential at the end of the inner field cage was accidentally set to a value $\sim 1.8 \%$ different from nominal, which led to a local distortion of the electrical field. A coarse voltage degrader was added on the inside of the IFC. It was segmented into five long sections, with potential steps of $\sim 2900 \mathrm{~V}$ from section to section. The main dimensions and HV settings of the TPC are listed in Table 2.

Table 2: Main dimensions and high-voltage settings of the TPC

\begin{tabular}{|l|c|}
\hline Outer diameter of inner field cage (IFC) & $106 \mathrm{~mm}$ \\
IFC wall thickness & $2 \mathrm{~mm}$ \\
IFC length & $786 \mathrm{~mm}$ \\
Inner diameter of outer field cage (OFC) & $816 \mathrm{~mm}$ \\
OFC wall thickness & $8 \mathrm{~mm}$ \\
OFC length & $2050 \mathrm{~mm}$ \\
Distance from high-voltage (HV) plane to cathode wire plane & $1557 \mathrm{~mm}$ \\
\hline HV on OFC membrane & $-17302 \mathrm{~V}$ \\
HV on IFC membrane & $-8623 \mathrm{~V}$ (nominal) \\
& $-8456 \mathrm{~V}$ (actual) \\
Electrical drift field & $111.1 \mathrm{~V} / \mathrm{cm}$ \\
\hline
\end{tabular}

Electrons from ionization induced by charged particles in the TPC gas drift upstream under the influence of the longitudinal electrical field; they are amplified and detected in the wire chamber. The tracking volume extends radially from $\sim 75 \mathrm{~mm}$ to $\sim 385 \mathrm{~mm}$ and over $\sim 1.5 \mathrm{~m}$ longitudinally.

Figure 3 shows the wire chamber before installation. The pad plane, mounted behind the Stesalit support structure with its six spokes, is clearly visible. It consisted of six identical printed circuit boards, with the readout pads toward the TPC volume, and the front-end electronics on the opposite side. Each sector comprised 662 pads of dimensions $6.5 \times 15 \mathrm{~mm}^{2}$ arranged in 20 concentrical rows, from $r=82.2 \mathrm{~mm}$ for the centre of the pads in the first row to $r=376.6 \mathrm{~mm}$ for those in the last row. The three wire planes (anode wires, cathode wires, and gating-grid wires) above the pad plane are hardly visible, though. The mechanical and electrical parameters of the wire chamber are listed in Table 3.

Table 3: Mechanical and electrical parameters of the wire chamber

\begin{tabular}{|l|c|c|c|c|}
\hline Item & $\begin{array}{c}\text { Distance from pad plane } \\
{[\mathrm{mm}]}\end{array}$ & $\begin{array}{c}\text { Size/diameter } \\
{[\mathrm{mm}]}\end{array}$ & $\begin{array}{c}\text { Pitch } \\
{[\mathrm{mm}]}\end{array}$ & $\begin{array}{c}\text { Potential } \\
{[\mathrm{V}]}\end{array}$ \\
\hline \hline Pad plane & 0 & $6.5 \times 15$ & $7 / 15.5$ & 0 \\
Anode wires & 5 & 0.020 & 4 & +1820 \\
Cathode wires & 10 & 0.070 & 2 & 0 \\
Gating wires & $15.75 / 16.25$ & 0.070 & $2(4 / 4)$ & $-67 \pm 35$ \\
\hline
\end{tabular}

The wire geometry is shown in Fig. 4. The pad plane and the cathode wires were at ground potential. With the anode wires at a potential of $+1820 \mathrm{~V}$ the nominal gas amplification was 


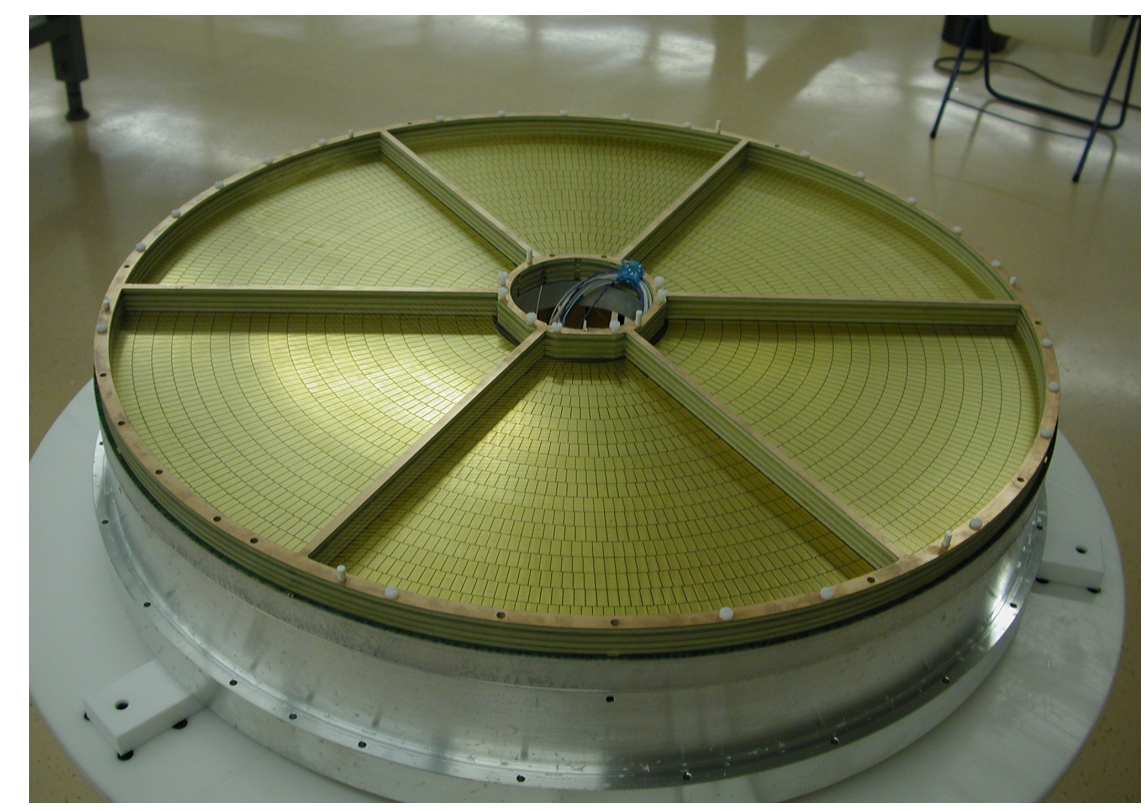

Fig. 3: Photograph of the TPC wire chamber before installation.

about $2 \times 10^{4}$. To prevent the ions from the gas amplification entering the drift volume a gating grid was placed $6 \mathrm{~mm}$ downstream of the cathode wire plane; its offset potential was $-67 \mathrm{~V}$. It consisted of two layers of wires at $0.5 \mathrm{~mm}$ distance in $z$, displaced with respect to each other by $2 \mathrm{~mm}$ radially. Each layer had a wire pitch of $4 \mathrm{~mm}$, giving an effective wire pitch of $2 \mathrm{~mm}$. To close the gating grid a voltage swing of $\pm 35 \mathrm{~V}$ on top of the offset potential was applied to the two wire planes.

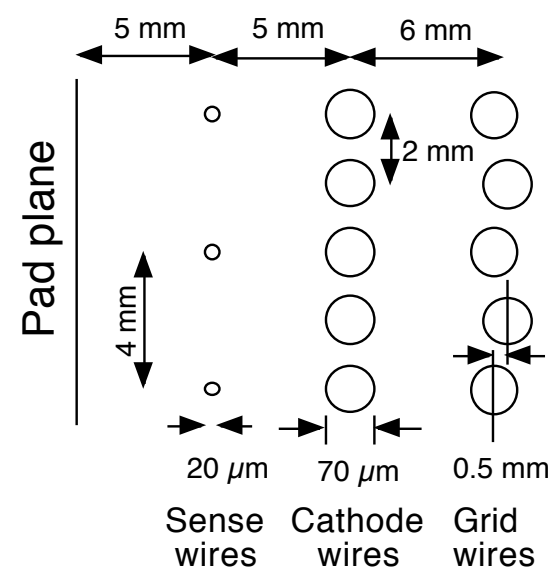

Fig. 4: The geometry of the chamber wires (not to scale).

This was the theory. The actual potentials were slightly different from the design values, with, however, serious effects for the electrical drift field close to the wire planes and the functioning of the gating grid. This will be discussed in Section 5.2. 


\subsection{Solenoidal magnetic field}

The magnet was operated at a current of $\pm 899.4 \mathrm{~A}$, with its polarity tied to the beam polarity ${ }^{2}$. It generated a magnetic field of $0.7 \mathrm{~T}$ parallel to the TPC axis. The actual field was mapped with a set of Hall probes prior to data-taking. These data served to tune a three-dimensional field simulation using the OPERA package ${ }^{3)}$. Figure 5 shows the longitudinal and radial components of the magnetic field in the TPC volume. Apart from the two 'saddles' at large radius ${ }^{4)}$, the field
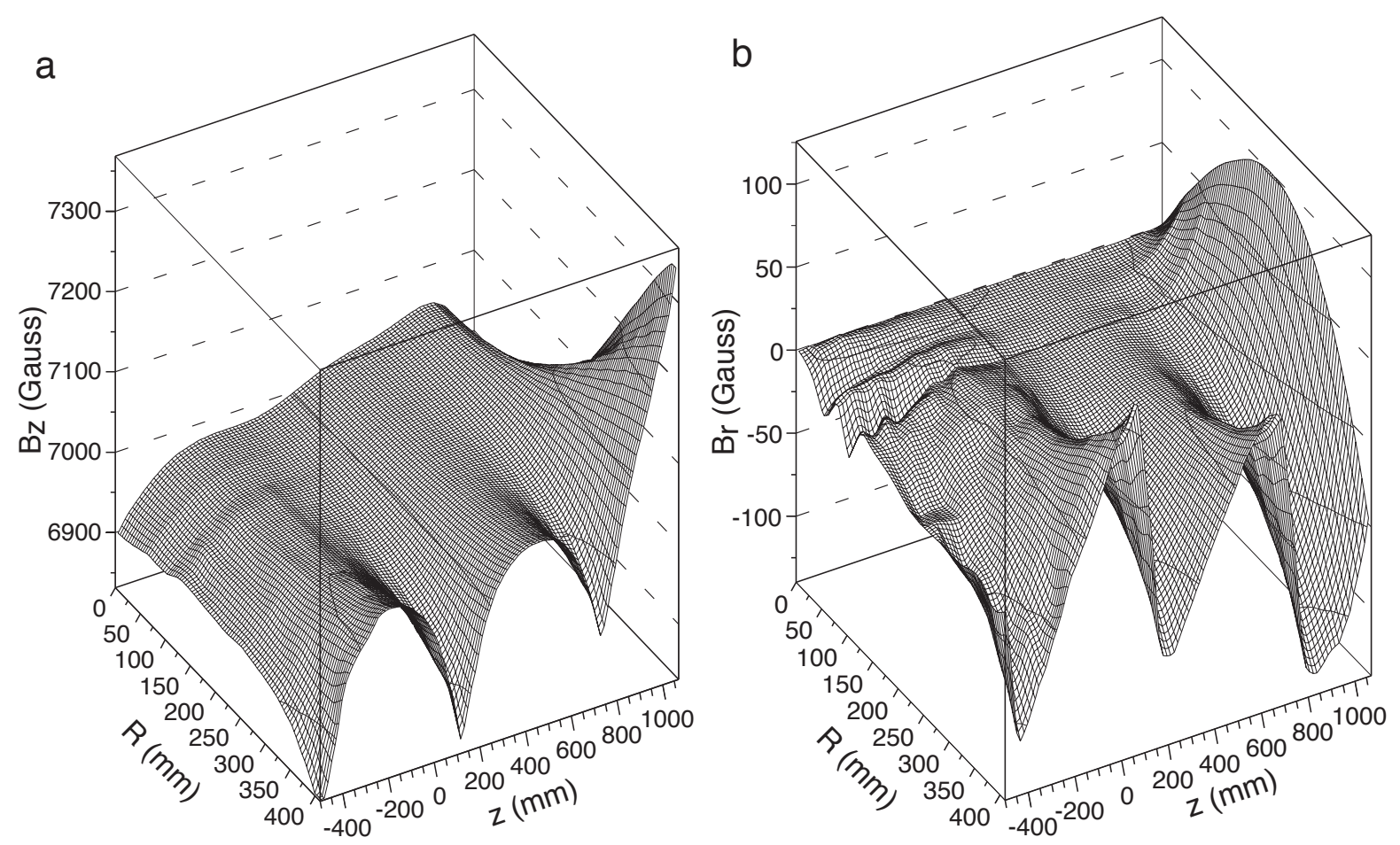

Fig. 5: Longitudinal (a) and radial (b) components of the solenoidal magnetic field as calculated by OPERA, which reproduced best the measured field map.

over most of the TPC volume is homogeneous within 1-2\%. Larger inhomogeneities of up to $\sim 5 \%$ are present at the downstream end of the TPC.

\subsection{Gas parameters}

The TPC was operated with the P9 gas mixture (91\% argon and 9\% methane by volume) at 5 mbar above atmospheric pressure. The gas flow varied between 60 and $120 \mathrm{l} / \mathrm{h}$, corresponding to a volume change every 12 to 6 hours. The atmospheric pressure and the ambient temperature in the experimental area were recorded over the full duration of data-taking. The temperature of the gas inside the TPC, however, was not measured. Taking into account that the TPC volume is thermally insulated, the gas temperature was determined from the temperature of the incoming fresh gas. The latter mixed almost instantaneously with the 'old' gas, leading to a homogeneous temperature over the full drift volume, but following, with some delay,

\footnotetext{
${ }^{2)}$ The HARP data-taking convention was that $B_{\mathrm{z}}>0$ refers to positive beam polarity.

3) Vector Fields Ltd, 24 Bankside, Kidlington, Oxford OX5 1JE, UK.

${ }^{4)}$ These are produced by two gaps in the coil structure leading to a local depletion of the magnetic field.
} 
variations in the ambient temperature in the hall ${ }^{5}$.

The dependences of the electron drift velocity and of the pad pulse height on temperature are illustrated in Fig. 6. The upper panel displays the day-night temperature variations in the experimental hall over 2.5 days of data taking in August 2002, and the temperature of the TPC gas as calculated from the gas flow and the hall temperature. The middle panel displays the target position reconstructed under the assumption of a constant drift velocity; clearly the actual drift velocity is in phase with the calculated TPC gas temperature. The lower panel displays the pad pulse height in terms of the specific ionization $\mathrm{d} E / \mathrm{d} x$; it is in phase with the hall temperature, since the amplification region in the TPC wire chamber follows the temperature of the pad plane which in turn is in phase with the hall temperature.

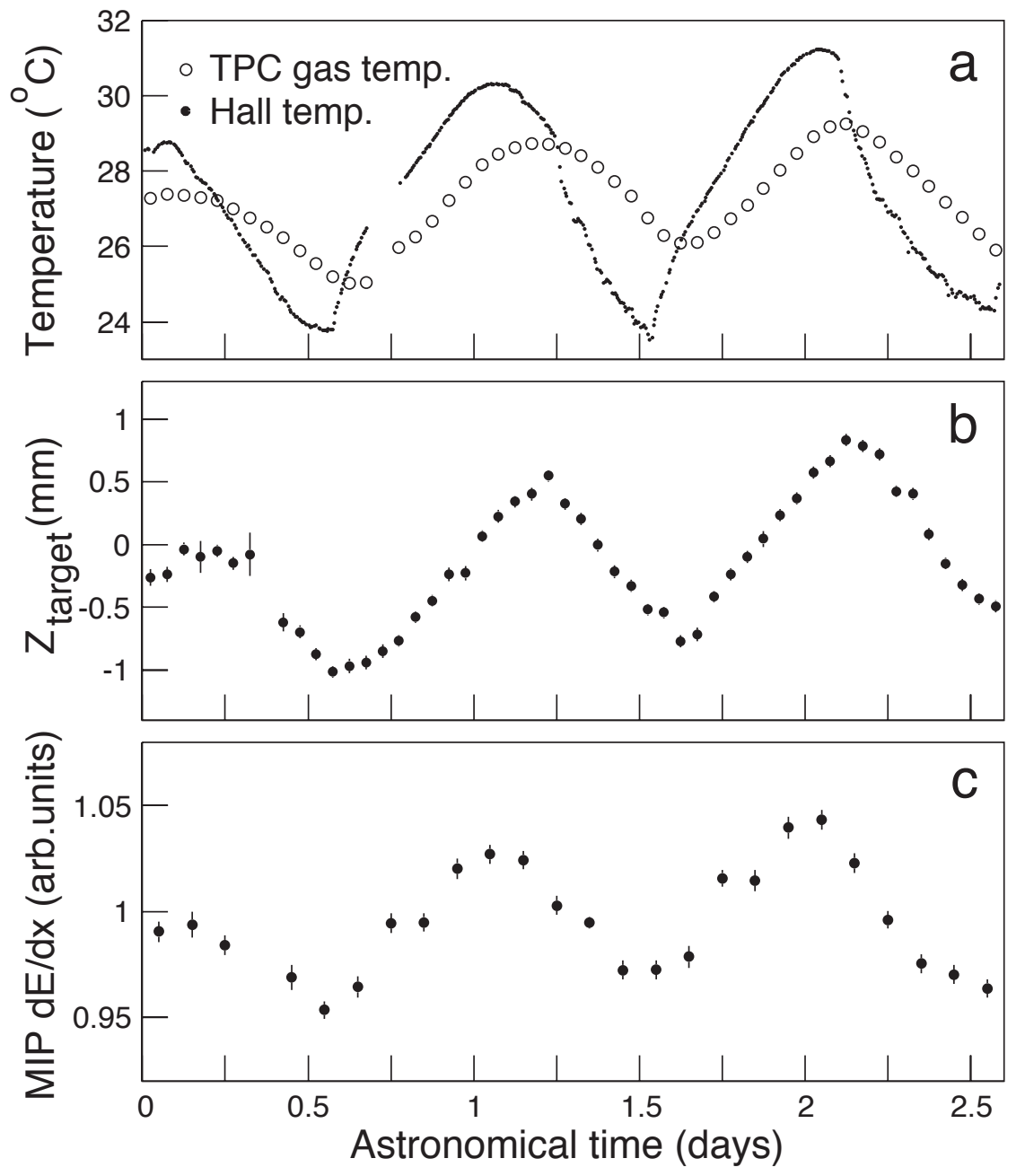

Fig. 6: (a) Hall temperature (black dots) and calculated gas temperature in the TPC (open circles) over a data-taking period of two and a half days; (b) variation of the reconstructed target position in $z$ under the assumption of a constant drift velocity; (c) variation of $\mathrm{d} E / \mathrm{d} x$.

Since the electron drift velocity depends on the electric field strength $E$ and the gas density

\footnotetext{
5) The incoming fresh gas was transported through non-insulated $\mathrm{Cu}$ lines, running in the experimental hall over a distance of $\sim 100 \mathrm{~m}$ from the supply to the TPC. Therefore, it enters the TPC at the temperature of the hall.
} 
$\rho$ only through the ratio $E / \rho$, we parametrize the temperature and pressure dependence of the electron drift velocity $v$ (for small variations) as

$$
\frac{\Delta v}{v}=-\alpha\left(\frac{\Delta P_{\mathrm{ATM}}}{P_{\mathrm{ATM}}}-\frac{\Delta T_{\mathrm{TPC}}}{T_{\mathrm{TPC}}}\right)
$$

where $T_{\mathrm{TPC}}$ denotes the absolute gas temperature in the TPC and $P_{\mathrm{ATM}}$ the absolute atmospheric pressure. We note that the same $\alpha$ also determines the dependence of the electron drift velocity on the electric field strength:

$$
\frac{\Delta v}{v}=\alpha \frac{\Delta E}{E}
$$

The value of $\alpha$ depends on the composition of the gas mixture ${ }^{6)}$. For the gas mixture used in 'thin $\mathrm{Be}+8.9 \mathrm{GeV} / c$ ' data taking, $\alpha$ has been measured according to Eq. (1) as

$$
\alpha=-0.27 \pm 0.01
$$

Altogether, the electron drift velocity depends on the electric field strength, temperature and atmospheric pressure, composition of the gas mixture, and humidity of the gas. It was determined for each data set separately by using the barrel RPCs as external reference, selecting tracks passing through the junctions of two neighbouring RPC pads in $z$. This method makes use of tracks in seven well-defined regions at large radius, exactly $240 \mathrm{~mm}$ apart along the $z$ direction, against which the track positions as reconstructed in the TPC can be compared ${ }^{7)}$. Table 4 gives the electron drift velocities for two physics data sets and for one cosmics data set, all taken during the year 2002, normalized to $25^{\circ} \mathrm{C}$ temperature, $966 \mathrm{hPa}$ pressure, and an electric field of $111.1 \mathrm{~V} / \mathrm{cm}$. There are differences between drift velocities larger than expected from the quoted statistical errors, likely to be caused by the use of different gas mixtures. There were also small variations of drift velocity between data sets taken with the same gas mixture, likely due to changes of gas humidity.

Table 4: Results for the electron drift velocity, normalized to $25^{\circ} \mathrm{C}$ temperature, $966 \mathrm{hPa}$ pressure and an electric field of $111.1 \mathrm{~V} / \mathrm{cm}$; the stated periods denote the respective use of batches of gas bottles; the quoted errors are statistical only.

\begin{tabular}{|l|c|c|c|}
\hline Period & $8.5 .-24.6 .02$ & $9.8 .-25.9 .02$ & 29.9.02-shutdown \\
\hline Data sets & Thin $\mathrm{Be}-8.0 \mathrm{GeV} / \mathrm{c}$ & Thin $\mathrm{Be}+8.9 \mathrm{GeV} / \mathrm{c}$ & calibration with cosmics \\
\hline \hline Drift velocity $[\mathrm{mm} / \mu \mathrm{s}]$ & $51.87 \pm 0.06$ & $52.19 \pm 0.06$ & $52.16 \pm 0.06$ \\
\hline
\end{tabular}

\footnotetext{
${ }^{6)}$ The gas was supplied from five different batches of gas bottles over the data-taking period in 2002; the supplier guaranteed the methane admixture as $(9 \pm 0.5) \%$ only.

${ }^{7)}$ Trying to extract the electron drift velocity from the reconstructed positions of a thin target and the end of the inner field cage resulted in a systematic bias of 1\%-2\%; according to Eq. (2), this bias is consistent with the static and dynamic distortions of the electrical field which tend to be largest at small radius (see Section 5.2).
} 


\subsection{Readout electronics}

The readout pads were connected to 4-channel preamplifier/shaper ASICs ${ }^{8)}$ soldered onto the upstream side of the wire chamber multilayer printed circuit board. The pre-amplified signals (FWHM 300 ns) were transmitted through pico-coaxial cables to 48-channel flash ADCs $(\text { FADC })^{9)}$ located a few metres from the TPC. The FADCs had a dynamic range of 10 bits and a sampling frequency of $10 \mathrm{MHz}$. In addition to the 300 samples corresponding to the total drift time in the TPC of $30 \mu \mathrm{s}$, pre- and post-samples were recorded for each trigger. Data were zero-suppressed at the level of the FADCs.

\section{FROM RAW DATA TO PHYSICS PARAMETERS}

\subsection{From time samples to cluster coordinates}

For each readout pad, contiguous non-zero pulse heights in $100 \mathrm{~ns}$ long time samples are assembled as 'hits', and combined with simultaneous hits in adjacent pads within the same pad row to 'clusters'. The radial coordinate $r$ of a cluster is the average radius of the pad row. The azimuthal coordinate $\phi$ of a cluster is calculated as the charge-weighted average of the $\phi$ positions of the cluster's three contiguous hits with the largest charge. The longitudinal $z$ coordinate of a cluster is derived from the charge-weighted average time of the cluster's largest hit ${ }^{10)}$.

\subsection{Pad pulse-height normalization}

To obtain the best $r \cdot \phi$ and $\mathrm{d} E / \mathrm{d} x$ resolutions, the TPC pads required a normalization of their pulse heights. This concerned common corrections for changes of gas gain with time, as well as pad-specific corrections for different intrinsic sensitivity.

The relative change of the gas gain at the sense wires is, for small variations of the gas density, proportional to the relative change of gas density:

$$
\frac{\Delta G}{G}=K \cdot \frac{\Delta \rho}{\rho}
$$

where $G$ is the gain at the sense wires, $\rho$ the gas density, and $K$ a proportionality constant. The constant $K$ is determined by the avalanche development in the vicinity of the sense wires. While the sign and the numerical value can be estimated, its precise value must be measured for every gas mixture and electric field configuration. It is in the range -3 to -8 for typical TPC gas mixtures [7]. The value for the HARP TPC was measured as $K \simeq-5.1$ and used to correct all pad pulse heights for gas density changes caused by changes of pressure and/or temperature (as discussed in Section 3.3, the relevant temperature variation is the one of the pad plane). After corrections, the stability of pad pulse heights was better than $\pm 4 \%$, both overall (determined from $\mathrm{d} E / \mathrm{d} x$ ) and pad by pad (determined from the minimum-ionizing pulse height) ${ }^{11)}$.

The pad-specific correction for different intrinsic sensitivity was achieved by calibrating the pad responses with the average ionization charge produced by ${ }^{83 \mathrm{~m}} \mathrm{Kr}$ decays ${ }^{12)}$. A gas of radioactive ${ }^{83 \mathrm{~m}} \mathrm{Kr}$ nuclei was produced by a ${ }^{83} \mathrm{Rb}$ source put into a bypass of the TPC input gas

\footnotetext{
8) ALCATEL SMB302.

${ }^{9)}$ The FADCs had been developed as prototypes for the ALICE TPC.

${ }^{10)}$ The $z$ position is calculated by multiplying the average time of the cluster's largest hit with the electron drift velocity, taking the latter's dependences according to Eqs. (1) and (2) on pressure, temperature and electric field strength into account.

${ }^{11)}$ This remaining $4 \%$ variation is attributed to variations of the gas mixture, varying humidity of the TPC gas, and to electronic drifts.

${ }^{12)}$ The method had been used before by ALEPH [8], DELPHI [9] and NA49 [10].
} 
line. The gas diffused into the TPC volume where the ${ }^{83 \mathrm{~m}} \mathrm{Kr}$ nuclei decayed with a lifetime of 1.86 hours. The decay products are mainly $\gamma$ rays. Owing to a high probability of internal $\gamma$ conversion and emission of Auger electrons, most of the $\gamma$ energy is transferred to electrons. These remained trapped in the TPC magnetic field and produced clusters of ionization charge which spread typically over 4 to 10 pads. The clusters' pulse-height spectrum with its characteristic peaks ranging from 9.4 to $41.6 \mathrm{keV}$ permitted in theory a very precise pad pulse-height normalization at the $1 \%$ level. In practice, cross-talk between neighbouring pads prevented that precision: cross-talk between pads in localized ${ }^{83 \mathrm{~m}} \mathrm{Kr}$ clusters modifies pad pulse heights differently from cross-talk between pads in clusters lined up along radial tracks. We come back to this in Section 5.1.

The procedure of pad-specific pulse-height normalization also allowed us to tag noisy and dead pads which were excluded from further analysis.

\subsection{From clusters to tracks}

The clusters constitute space points along the track trajectory. Each space point has three uniquely determined coordinates: $r, \phi$, and $z$. Pattern recognition is facilitated since no ghost solutions need to be eliminated.

The pattern recognition must deal with different track types: high- $p_{\mathrm{T}}$ and low- $p_{\mathrm{T}}$ tracks originating from the target, beam muons, and cosmic muons. Our pattern recognition of tracks with $p_{\mathrm{T}} \geq 0.05 \mathrm{GeV} / c$ originating from the target region, and of cosmic muons, is based on the TOPAZ histogram technique [11]: a 2-dimensional histogram of the ratio $z / r$ against azimuthal angle $\phi$ is filled with all reconstructed clusters. Physical tracks will populate one or few adjacent bins (the bin sizes are suitably chosen) and thus are easily recognised.

We have determined the overall reconstruction efficiency as $95 \pm 1 \%$ (for tracks with $\left|p_{\mathrm{T}}\right|>$ $0.1 \mathrm{GeV} / c$ and $20^{\circ}<\theta<135^{\circ}$ ) by extensive eye-scanning of events, where the $1 \%$ error reflects the variation between different data sets.

\subsection{Helix fit of tracks}

Before we discuss the errors of cluster coordinates in Sections 4.5 and 4.7, we address the problem of how to fit data points with three coordinates, the errors of all of which need to be taken properly into account.

Conventional fit algorithms of multidimensional data points have only one coordinate with error (referred to as 'dependent' variable) while all other coordinates (referred to as 'independent' variables) are error-free.

In the ALEPH TPC, for example, first a circle was fitted in the transverse $x-y$ plane using a modified Chernov-Ososkov least-squares algorithm [12]. The modification concerned the introduction of a 'weight' for each data point derived from the $r \cdot \phi$ error. In a second step a straight line was fitted in the $s-z$ plane, where $s$ denotes the transverse arc length from the origin to the data point. While this approach is perfectly adequate for high-momentum tracks, it becomes incorrect for strongly bent tracks where two independent coordinate errors can no longer be combined into one single 'weight'. Since in the HARP energy range low-momentum tracks are quite common, we adopted for the fit of trajectories in the HARP TPC the 'generalized least-squares fit' concept. This is the formal generalization of the standard least-squares fit for an arbitrary number of error-prone dimensions, and the solution of the equations resulting from the $\chi^{2}$ minimization with the Lagrange multiplier method. The mathematical intricacies can be found in Ref. [13]. 
For the three parameters that describe the circle projection of a helix, we adopted the TOPAZ parametrization [14] for the attractive feature of avoiding any discontinuity in the numerical values of fit parameters. Most importantly, it permits a smooth transition between charge signs of a track. For more details on the parametrization and the fit procedure, we refer to Ref. [15].

The helix fit yields in one single step the transverse momentum $p_{\mathrm{T}}$, the charge sign, and the polar angle $\theta$ of a track.

\subsection{Azimuthal coordinate precision}

The width of $6.5 \mathrm{~mm}$ of the pads was chosen close to the $6.2 \mathrm{~mm}$ used in the ALEPH TPC $[8,16]$, and so was the distance between pad and sense wire planes $(5 \mathrm{~mm}$ compared with $4 \mathrm{~mm}$ in ALEPH). This ensured a reasonably good knowledge of the pad response function and the assurance that the set goal in $\sigma_{r \phi}$ was realistic.

In this section, we discuss the statistical effects that contribute to the observed $r \cdot \phi$ resolution of clusters. The relevant geometric dimensions and angles are depicted in Fig. 7. The square

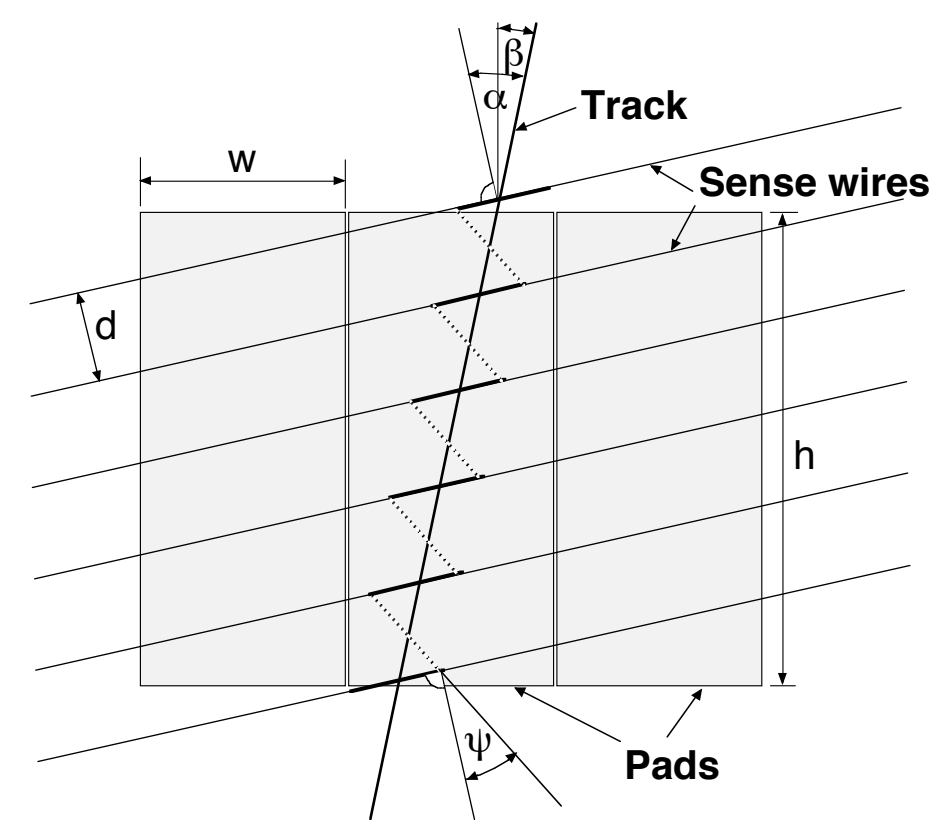

Fig. 7: Particle track across pads: definition of geometrical dimensions and of the angles $\alpha, \beta$, and $\psi$.

of the resolution $\sigma_{r \phi}$ is given by the quadratic sum of $r \cdot \phi$ resolutions from various statistical or quasi-statistical fluctuations:

$$
\begin{aligned}
\sigma_{r \phi}^{2}= & \frac{\kappa^{2}}{12}(\tan \alpha-\tan \psi)^{2} \frac{d^{2}}{h N_{\mathrm{pc}}} \cos \beta \cos ^{2}(\alpha-\beta) \\
& +\frac{2 L}{v_{\mathrm{el}}} \frac{D(0)}{C+(\omega \tau)^{2}} \frac{1}{h N_{\mathrm{el}}} \cos \beta \cos ^{2}(\alpha-\beta) \\
& +\frac{\kappa^{2}}{12} \frac{h}{N_{\mathrm{pc}}} \cos \beta \tan ^{2} \beta \\
& +\sigma_{\mathrm{dig}}^{2}+\sigma_{\text {ped }}^{2}+\sigma_{\text {equ }}^{2}+\sigma_{\text {Xtalk }}^{2}+\sigma_{\text {3pad }}^{2} .
\end{aligned}
$$

With a view to avoiding undue complication, the 'stiff-track approximation' is made in Eq. (4): the angle $\beta$ between the track and the pad orientation is small $\left(\beta \leq 10^{\circ}\right.$, i.e., tracks traverse the 
pad rows nearly perpendicularly). Furthermore, tracks are supposed to be parallel to the pad plane, i.e., the dependence of the $r \cdot \phi$ resolution on the polar-angle $\theta$ is ignored. We proceed with discussing each term in turn.

If a track crosses the sense wires not perpendicularly but under an angle $\alpha$ (in the HARP TPC, $\alpha$ ranges for a radial track with $\beta=0^{\circ}$ from $-30^{\circ}$ to $+30^{\circ}$ ), the $r \cdot \phi$ resolution deteriorates because of the fluctuation in the number of primary electron clusters $N_{\mathrm{pc}}$ and in their 'cluster size'. To this 'wire angular effect' the 'wire $E \times B$ effect' adds linearly. Together, they give rise to the first term in Eq. (4). With $\kappa=1.5$ (which takes into account the cluster size distribution), $N_{\mathrm{pc}}=2.6 \mathrm{~mm}^{-1}$ (the number of primary electron clusters per unit length), and the effective Lorentz angle ${ }^{13)}$ near the sense wires of $\psi=16^{\circ}[17,18]$, the contribution from the angular and the wire $E \times B$ effects ranges between zero for $\alpha=\psi=16^{\circ}, 70 \mu \mathrm{m}$ for $\alpha=30^{\circ}$, and $200 \mu \mathrm{m}$ for $\alpha=-30^{\circ}$. This asymmetric $r \cdot \phi$ resolution holds for $B>0$; for $B<0$ the angles $\alpha$ change sign.

The second term in Eq. (4) refers to the transverse diffusion of electrons over the length $L$. At a magnetic field of $0.7 \mathrm{~T}$, the transverse diffusion constant for an electron is reduced by the factor $1 /\left(C+(\omega \tau)^{2}\right)$ with respect to the one at zero magnetic field, where $C=2.8 \pm 0.2$ and $\omega \tau$ $=3.28 \pm 0.07$ [19]. With the number of electrons per unit length $N_{\mathrm{el}}=100 \mathrm{~cm}^{-1}$, an electron drift velocity $v_{\mathrm{el}}=5 \mathrm{~cm} / \mu \mathrm{s}$, and a diffusion constant $D(0)=0.9 \mathrm{~m}^{2} / \mathrm{s}$ [20], the $r \cdot \phi$ resolution from transverse diffusion is $130 \mu \mathrm{m}$ for a drift length of $L=1 \mathrm{~m}$.

The third term in Eq. (4) refers to the 'pad angular effect'. This term contributes only for low-momentum tracks which have a non-zero pad crossing angle $\beta$. For $\beta=10^{\circ}$, the contribution to the $r \cdot \phi$ resolution is $190 \mu \mathrm{m}$.

The term $\sigma_{\text {dig }}$ denotes the $r \cdot \phi$ fluctuation which stems from the sampling of the pulse height (not of the integral charge of the time slice) of the pad signals every $100 \mathrm{~ns}$. Its contribution is estimated at $140 \mu \mathrm{m}$. The term $\sigma_{\text {ped }}$ reflects pedestal variations (including electronics noise) and losses from ADC thresholds. Its size is estimated at $70 \mu \mathrm{m}$. The term $\sigma_{\text {equ }}$ stems from the uncertainty of the equalization of the pedestal pulse heights (including preamplifier nonlinearities) achieved in the calibration with ${ }^{83 \mathrm{~m}} \mathrm{Kr}$ decays. Its size is estimated at $110 \mu \mathrm{m}$.

Once again, this was the theory, and the above-listed fluctuations rather reflect academic interest. In practice, the $r \cdot \phi$ resolution was deteriorated by electronics cross-talk between pad signals. The consequences of this cross-talk are represented by $\sigma_{\mathrm{Xtalk}}$ and $\sigma_{3 \mathrm{pad}}$ in Eq. (4). We come back to this in Section 5.1.

The above-discussed error sources are summarized in Table 1.

\section{6 $\quad p_{\mathrm{T}}$ resolution and charge identification}

With the $r \cdot \phi$ resolution of $360 \mu \mathrm{m}$ as stated as objective in Table 1 , and an average of 13 clusters per track ${ }^{14)}$, the resolution $\sigma\left(1 / p_{\mathrm{T}}\right)$ would have been $0.18(\mathrm{GeV} / c)^{-1}$. The cross-talk between pad signals, however, prevented such good performance. When treating the effect of cross-talk as an additional quasi-statistical $r \cdot \phi$ fluctuation, a $\sigma\left(1 / p_{\mathrm{T}}\right)$ in the range $0.45-0.50(\mathrm{GeV} / c)^{-1}$ is expected with the observed $r \cdot \phi$ resolution of clusters. As stated in Table 1, the latter ranges from $600 \mu \mathrm{m}$ for 3-pad clusters to $2.4 \mathrm{~mm}$ for 1-pad clusters, with an effective average of $\sim 1.0 \mathrm{~mm}$.

\footnotetext{
${ }^{13)}$ The tangent of the Lorentz angle is proportional to the magnetic $B$ field and changes sign with its polarity.

14) Because of lower systematic precision of the $\phi$ coordinate of clusters in pad rows 1 and 20, next to the inner and outer sector borders, those clusters were routinely omitted in track fits; a further reduction in the number of clusters per track arose from cuts on cluster quality, from losses due to dead regions, and from the requirement of minimum distance to dead pads.
} 
This $p_{\mathrm{T}}$ resolution is significantly improved when using the position of the beam point as an additional point along the trajectory, primarily because of the significantly increased lever arm. The beam point is known from the extrapolation of the trajectory of the incoming beam particle. Its transverse error comprises three error sources. The first is from the extrapolation error of the beam trajectory (which is determined by three small multiwire proportional chambers located in the beam line), the second stems from multiple scattering of the beam particle. Excluding the target itself, the transverse error at $z=0$ is $0.46 \mathrm{~mm}$ from extrapolation, and $0.51 \mathrm{~mm}$ from multiple scattering of a $3 \mathrm{GeV} / c$ beam particle.

Since a secondary track loses energy by ionization in the target and in materials between the target and the TPC volume, a correction must be calculated which moves the real beam point to a 'virtual' beam point which is bias-free with respect to the extrapolation of the track's helical trajectory measured in the TPC ${ }^{15}$ ) At the same time, the transverse error of the virtual beam point stemming from multiple scattering of the secondary track must be added as third error to the two errors from the beam particle discussed above. To give a numerical example: for a pion emitted from a thin Be target at $\theta=45^{\circ}$ and with a momentum of $200 \mathrm{MeV} / c$ measured in the TPC, the virtual beam point is shifted in the transverse direction by $0.05 \mathrm{~mm}$ from the real beam point, and the transverse error from multiple scattering is $1.0 \mathrm{~mm}$ (for a proton these numbers change to $0.7 \mathrm{~mm}$ and $2.5 \mathrm{~mm}$, respectively).

With a transverse precision of $1 \mathrm{~mm}$ of the beam point, a resolution of $\sigma\left(1 / p_{\mathrm{T}}\right)$ in the range $0.20-0.25(\mathrm{GeV} / \mathrm{c})^{-1}$ is achieved, i.e., an improvement by approximately a factor of two with respect to $0.45-0.50(\mathrm{GeV} / \mathrm{c})^{-1}$. This improvement in precision makes very clear, though, that great care must be taken to correctly calculate the transverse precision of the virtual beam point, separately for every track.

For low beam and/or secondary track momenta the beam point is less precise and the improvement of $p_{\mathrm{T}}$ resolution is less pronounced.

With the use of the beam point in the track fit, the charge misidentification probability at $p_{\mathrm{T}}=2 \mathrm{GeV} / c$ is at the $2 \%-3 \%$ level, taking a non-Gaussian tail into account.

\subsection{Longitudinal coordinate precision and polar-angle resolution}

With an amplitude sampling every $100 \mathrm{~ns}$, a preamplifier transfer function with a FWHM of $\sim 300 \mathrm{~ns}$, a fixed amplitude threshold to mark the start and the stop of a pulse-train, and an amplitude-weighted average as estimate of the $z$ position of a cluster, a resolution in the longitudinal coordinate of $\sim 2.6 \mathrm{~mm}$ is expected. In comparison, the contribution from longitudinal diffusion is with $330 \mu \mathrm{m}$ over a drift distance of $0.5 \mathrm{~m}$ negligible. The expected polar-angle resolution (without beam point and hence without deterioration from multiple scattering in materials before the particle enters the TPC) is $6.6 \mathrm{mrad}$ for a polar angle of $60^{\circ}$.

This performance figure does not take into account the additional contribution from the electronics cross-talk between pad signals of comparable magnitude (see Table 1 and Section 5.1).

\section{8 $\mathrm{d} E / \mathrm{d} x$ resolution}

With a view to achieving the best possible $\mathrm{d} E / \mathrm{d} x$ resolution, it is common to order the pulseheight samples along a track according to pulse height, and to discard fixed fractions of all samples at both the low and the high end. The relatively small number of samples in the HARP TPC (maximum of 20) did not permit this procedure; rather the two samples with the largest

\footnotetext{
${ }^{15)}$ The elimination of the bias from energy loss with the 'virtual beam point' concept is motivated by its easy implementation in the 'generalized least-squares' fit.
} 
pulse heights were removed. Samples from pad rows 1 and 20 (which had systematically lower pulse heights) were ignored throughout. A minimum of five samples for calculating $\mathrm{d} E / \mathrm{d} x$ was required. The standard analysis cut for a 'good' $\mathrm{d} E / \mathrm{d} x$ required a minimum of eight samples after removal of the two samples with the largest pulse heights.

General experience with the measurement of energy loss, and specific experience from the ALEPH TPC has led to the following empirical expectation for the resolution [16]:

$$
\sigma / E_{\mathrm{loss}} \simeq G \cdot N^{-0.5} \cdot(\sin \theta)^{0.4}
$$

where $N$ is the number of samples and $\theta$ is the track's polar angle; $G$ is a proportionality constant which is 0.68 for HARP.

With $N=18$ and $\theta=75^{\circ}$, the expected resolution $\sigma / E$ from Eq. (5) is $16 \%$ for minimumionizing particles (the track length would correspond to $\sim 300 \mathrm{~mm}$ ).

The removal of the two samples with the largest pulse heights leads to a small, though significant, dependence of $\mathrm{d} E / \mathrm{d} x$ on the polar angle $\theta$ (because of the requirement of a fixed minimum pulse height in each pad to cross the threshold for recording). This polar-angle dependence was parametrized and corrected for.

Figure 8 shows the distribution of the energy loss measured in the HARP TPC, after all corrections, normalized to $25^{\circ} \mathrm{C}$ temperature and $938 \mathrm{hPa}$ pressure of the TPC gas. Negative pions in the momentum band from $0.45-0.80 \mathrm{GeV} / c$ were selected. The track length along samples with non-zero pulse height was required to be in the range $200-400 \mathrm{~mm}$. The Gaussian fit of the distribution gives a resolution ${ }^{16)}$ of $16 \%$.

The agreement between the measured and the expected resolution suggests that remaining cross-talk (see Section 5.1) is not detrimental for $\mathrm{d} E / \mathrm{d} x$.

\section{RECOVERY FROM TRACK DISTORTIONS}

The coordinates of TPC clusters were affected by two unforeseen phenomena: electronics crosstalk from outputs to inputs of preamplifiers of pad signals, and distortions of the electric and magnetic fields in the TPC volume. While the field distortions were eventually well understood and adequately corrected by appropriate software, the effects from cross-talk could be corrected only in part. The remaining footprints from cross-talk constitute the limitation in the precision of the $\phi$ coordinate of clusters.

\subsection{Distortions from electronics cross-talk}

The preamplifier ASICs were soldered onto the upstream face of the multilayer motherboard which features the readout pads at the downstream face. Because of a fabrication error, some interconnection pins between layers were too long and caused capacitive coupling from preamplifier outputs to the inputs of the same or of other preamplifiers. This resulted in three crosstalk types: unidirectional, bidirectional, and self cross-talk, shown schematically in Fig. 9. The level of cross-talk was dependent on the size of the coupling capacity which varied in a rather erratic fashion (the six TPC sectors, although identical in design, had different cross-talk levels). Bidirectional cross-talk is special in the sense that there is an instability limit above which the channel oscillates: that explained in retrospect the unexpectedly large fraction of 'noisy' channels encountered. Self cross-talk is also special since it cannot be corrected and must be considered an intrinsic feature of the preamplifier concerned.

\footnotetext{
${ }^{16)}$ The resolution is determined in a Gaussian fit where the fit range is truncated at the side of large pulse heights at half maximum.
} 


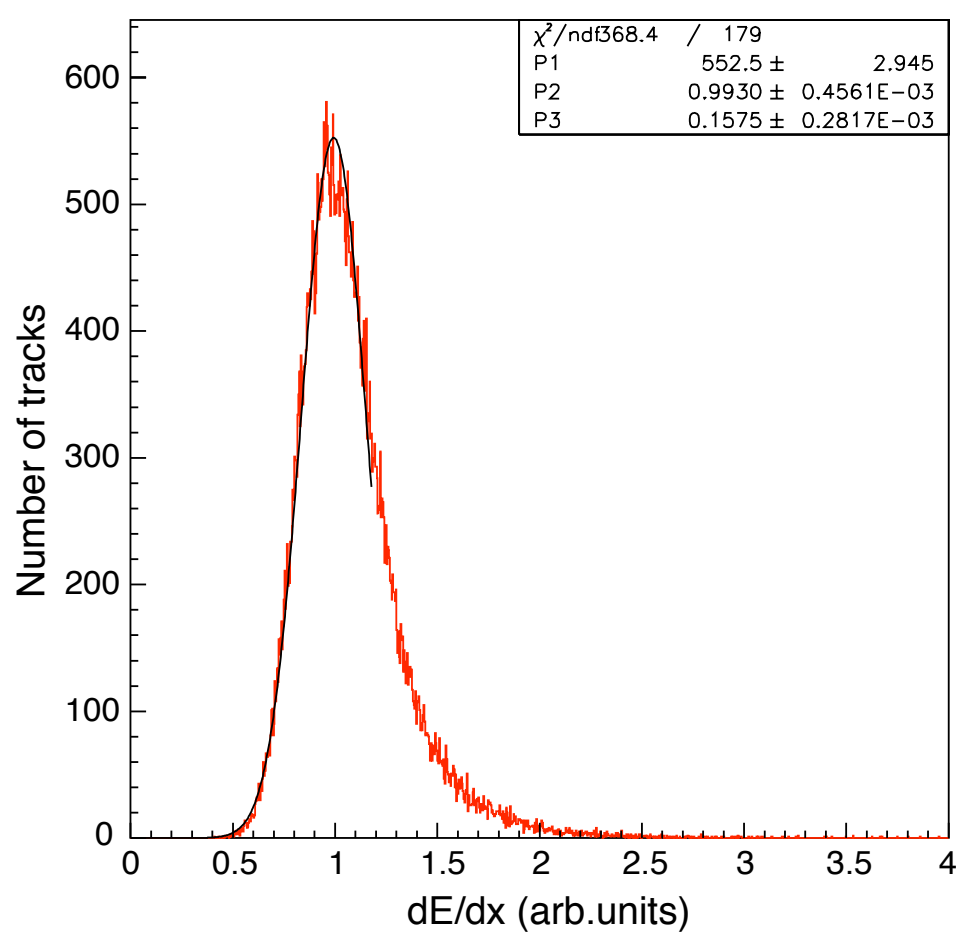

Fig. 8: $\mathrm{d} E / \mathrm{d} x$ distribution (arb. units), averaged over all six TPC sectors; overlayed is a Gaussian fit that is truncated at the side of large pulse heights at half maximum.

In uni- and bidirectional cross-talk pulse differentiation takes place, by contrast with self cross-talk which features pulse integration. Isolated cases of one type of cross-talk were the exception, the rule was rather the superposition of two or of all three types. Another important aspect for cross-talk correction algorithms was that the preamplier inverted the intrinsically positive signal polarity. The FADCs were designed to digitize signals of negative polarity only, hence the positive overshoot from differentiation was not recorded.

This complexity led us to adopt a pragmatic approach. Into every pad a known test charge was injected and all signals in the same and in any other pad were recorded. Then a correction algorithm was constructed which allowed the suppression of unwanted 'satellite' signals caused by cross-talk. This algorithm employs measured signals only and hence is restricted to negative pulse heights. It does not correct for the reduction of pulse height by positive overshoot on top of a negative pulse height.

The net effect of cross-talk for a specific pad in a cluster is a quasi-statistical fluctuation of its pulse height. This fluctuation amounts to $\sim 25 \%$ and leads to an average contribution to the $r \cdot \phi$ resolution of $530 \mu \mathrm{m}$ (the weighted average between zero and the maximum of $2000 \mu \mathrm{m}$ listed in Table 1). This contribution to the $r \cdot \phi$ resolution is referred to as $\sigma_{\mathrm{Xtalk}}$ in Eq. (4).

As a further consequence of cross-talk, we limited the number of pads in a cluster that are used to calculate the $\phi$ position as the weighted average of the geometrical pad centres, to maximally three contiguous pads with the largest pulse height ${ }^{17)}$. The contribution from this limitation to the $r \cdot \phi$ resolution is $110 \mu \mathrm{m}$ for 3 -pad clusters. More serious is that, in quite a

\footnotetext{
17) The pad response function itself that is well represented by a Gaussian with $\sigma=4.5 \mathrm{~mm}$, would call for the weighted average of up to five pads per cluster.
} 


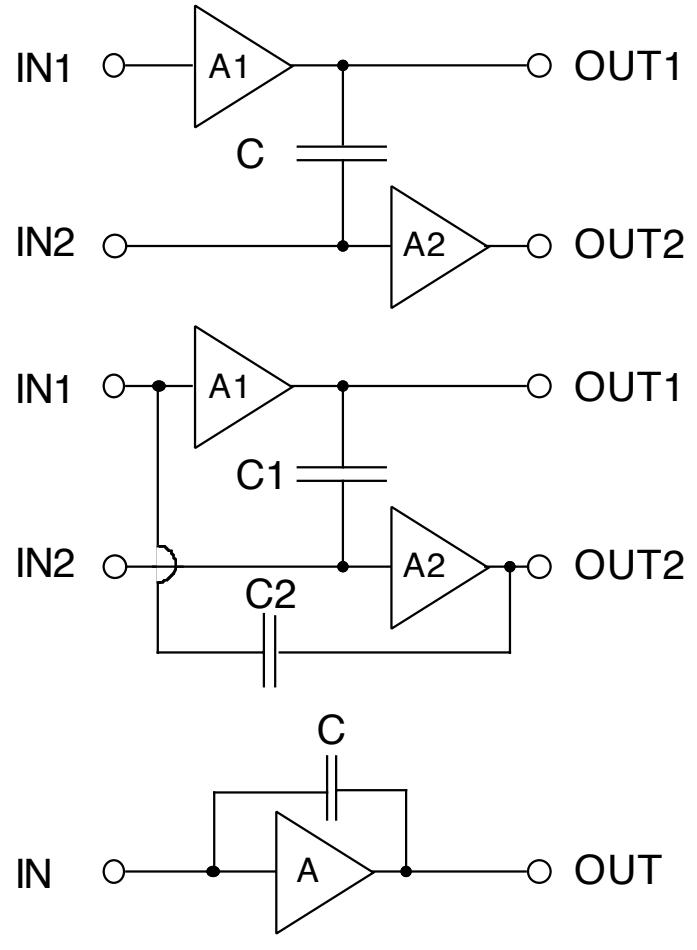

Fig. 9: The three cross-talk types: unidirectional (top), bidirectional (middle), and self cross-talk (bottom).

number of cases, pad signals fell below threshold, leading to 2-pad and even 1-pad clusters. In the 1-pad case, the resolution is with $\sim 2 \mathrm{~mm}$ rather poor ${ }^{18)}$. This contribution to the $r \cdot \phi$ resolution is referred to as $\sigma_{3 \text { pad }}$ in Eq. (4).

Adding quadratically the statistical and quasi-statistical resolutions gives an effective average of $\sim 1.0 \mathrm{~mm}$ for the overall $r \cdot \phi$ resolution (weighted according to the relative population of 1-pad, 2-pad and 3-pad clusters), which reflects the dominance of of uni- and bidirectional cross-talk over the intrinsic limitations discussed in Section 4.5.

Analogously with uni- and bidirectional cross-talk for the $r \cdot \phi$ resolution, self cross-talk deteriorates the $z$ resolution and, as a consequence, the polar-angle resolution (see Table 1). Yet the worse polar-angle resolution remains good enough not to deteriorate appreciably the momentum resolution when calculating the momentum from $p=p_{\mathrm{T}} / \sin \theta$.

\subsection{Distortions from field inhomogeneities}

The operation of the TPC requires nearly perfectly parallel longitudinal electric and magnetic field vectors. Unwanted transverse field components cause track distortions. The effects are determined by the Langevin equation (see, for example, Ref. [7]) which gives the drift vector $\vec{v}$ of electrons in the electric field $\vec{E}$ and magnetic field $\vec{B}$ of the TPC:

$$
\vec{v}=-\frac{\mu}{1+(\omega \tau)^{2}}\left(\vec{E}-\frac{\omega \tau}{|\vec{B}|}(\vec{E} \times \vec{B})+\frac{(\omega \tau)^{2}}{|\vec{B}|^{2}} \vec{B}(\vec{E} \cdot \vec{B})\right)
$$

\footnotetext{
${ }^{18)}$ Because of the origin of the appearance as 1-pad cluster, the $r \cdot \phi$ resolution of 1-pad clusters is not the pad width divided by $\sqrt{12}$.
} 
where $e$ and $m$ are the electric charge and the mass of the electron; $\mu$ denotes the electron mobility, i.e., its velocity per unit electric field strength in the absence of a magnetic field; and $\omega \tau=3.28 \pm 0.07$ is the product of the electron's cyclotron frequency and the average time between successive collisions with gas molecules.

The approximate displacements in $r$ and $r \cdot \phi$ that arise from a small transverse component of the magnetic field, for a perfectly aligned electric field $\vec{E}=\left(0,0, E_{\mathrm{z}}\right)$ and a (nearly) parallel magnetic field $\vec{B}=\left(B_{\mathrm{r}}, B_{r \phi}, B_{\mathrm{z}}\right)$, are (for details, see Refs. [21] and [22]):

$$
\begin{aligned}
\Delta_{\mathrm{r}} & \simeq-L \frac{1}{1+(\omega \tau)^{2}}(\omega \tau)^{2} \alpha_{\mathrm{r}}^{\mathrm{m}} \\
\Delta_{r \phi} & \simeq-L \frac{1}{1+(\omega \tau)^{2}}\left(\mp \omega \tau \alpha_{\mathrm{r}}^{\mathrm{m}}\right),
\end{aligned}
$$

with the angle $\alpha_{\mathrm{r}}^{\mathrm{m}}=B_{\mathrm{r}} / B_{\mathrm{z}}$, and the drift length $L(L>0)^{19)}$.

The distortions from small transverse magnetic field components are in the $r$ coordinate larger by a factor of $\omega \tau$ than those in the $r \cdot \phi$ coordinate.

The approximate displacements in $r$ and $r \cdot \phi$ that arise from a small transverse component of the electric field, for a perfectly aligned magnetic field $\vec{B}=\left(0,0, B_{\mathrm{z}}\right)$ with $B_{\mathrm{z}}>0$ and a (nearly) parallel electric field $\vec{E}=\left(E_{\mathrm{r}}, E_{r \phi}, E_{\mathrm{z}}\right)$, are:

$$
\begin{aligned}
\Delta_{\mathrm{r}} & \simeq-L \frac{1}{1+(\omega \tau)^{2}} \alpha_{\mathrm{r}}^{\mathrm{e}} \\
\Delta_{r \phi} & \simeq-L \frac{1}{1+(\omega \tau)^{2}}\left( \pm \omega \tau \alpha_{\mathrm{r}}^{\mathrm{e}}\right)
\end{aligned}
$$

with the angle $\alpha_{\mathrm{r}}^{\mathrm{e}}=E_{\mathrm{r}} / E_{\mathrm{z}}$. The distortions because of small transverse electric field components are in the $r \cdot \phi$ coordinate larger by a factor of $\omega \tau$ than those in the $r$ coordinate, opposite to the case of the magnetic field inhomogeneity.

A characteristic feature of track distortions from transverse inhomogeneities of both the magnetic and electric field is that the $r \cdot \phi$ distortions change sign with the magnetic field polarity.

The HARP TPC suffered from an inhomogeneity of the magnetic field-unavoidable from mechanical constraints - and several unplanned inhomogeneities of the electric field. We distinguish between 'static' distortions that are constant and present all the time, and 'dynamic' distortions that show up only during the $400 \mathrm{~ms}$ long accelerator spill ${ }^{20)}$. A careful study of track distortions permitted a nearly perfect reconstruction of the field inhomogeneities, which resulted in correction maps for the $r$ and $\phi$ coordinates of clusters for every point inside the TPC volume.

For a bias-free measurement of track distortions, reference to an external coordinate system must be made. Fortunately, this was provided for at large radius by $\sim 16 \mathrm{~mm}$ wide overlaps ${ }^{21)}$ of the barrel RPCs [6], and - for physics tracks - at small radius by the beam point. For throughgoing cosmic-muon tracks that pass close to the TPC axis, the closest point of approach to the

\footnotetext{
${ }^{19)}$ Here, and elsewhere in this paper, whenever there is a double sign the upper sign refers to the magnetic field orientation $\vec{B}=\left(0,0, B_{\mathrm{z}}\right)$ with $B_{\mathrm{z}}>0$, and the lower sign to the opposite magnet polarity.

${ }^{20)}$ The 'margaritka' effect, discussed below, is present at any time, but it is significantly stronger during the accelerator spill.

${ }^{21)}$ The $\sim 16 \mathrm{~mm}$ width permitted the precise measurement of distortions when averaged over many tracks.
} 
TPC axis was used as small-radius reference point: when fitting such through-going cosmicmuon tracks, distortion effects in the opposite hemispheres will cancel in linear approximation; hence the closest point of approach to the TPC axis is independent of the distortions and therefore constitutes an unbiased reference point at small radius.

The study of through-going cosmic-muon tracks which passed close to the TPC axis permitted the understanding of static distortions, while the study of physics tracks permitted the understanding of dynamic distortions. The distortions were measured in the following way. First, the track's curvature was estimated from a fit of the (distorted) track trajectory. With this curvature, a reference trajectory through the unbiased reference points at large and small radius was constructed which served to determine the distortions of clusters in the $r \cdot \phi$ coordinate.

There is an important difference between the measurements of static and dynamic distortions. In the measurement of static distortions with through-going cosmic muons which pass close to the TPC axis, the muon track's curvature is unbiased thanks to the cancellation of distortions in opposite hemispheres. For physics tracks this cancellation is absent since they involve one hemisphere only. Therefore, the initial estimate of the physics track's curvature, and hence the initial estimate of dynamic distortions, is strongly biased in the case of large distortions. This bias was eliminated by an iterative procedure.

The reference to the external coordinate system that is provided by the RPC overlaps is possible for every data set; this turned out to be of decisive importance since dynamic field distortions have different characteristics in different data sets. We stress that a fit of the clusters along a track without reference to an external coordinate system is not an adequate tool to study distortions, since the fit tends to 'co-move' with the distortions.

Thanks to the different dependences of different types of distortions on $r$ and $z$, on event time, and on the polarity of the magnetic field, it was possible to isolate them from each other and to determine their individual strengths from a fit to the data. This task was complicated by correlations of different strengths between different types of distortions. Yet what matters is that the overall sum of the effects from various types of distortions represents a model that reproduces the observed distortions with adequate precision. The proof of the model's success is the demonstration that in every volume element of the TPC, at any time in the spill, track distortions are compatible with zero after correction with the model's prediction.

Our modelling of track distortions needs an apparent $2 \mathrm{~mm}$ azimuthal shift of the RPC overlaps in the counter-clockwise direction when looking downstream, for positive magnet polarity. This shift needs to be considered equally in static and dynamic distortions. It is not related with the mechanical position of the RPCs since it changes sign with the magnetic field. We conjecture that this apparent azimuthal shift stems from the correlations between the various types of static distortions that we found necessary to take into account. Since this azimuthal shift of the RPC overlaps is equivalent to a small rotation of the coordinate system, reconstructed physics quantities are not affected.

We discuss below the recovery from static and dynamic distortions. A detailed account can be found in Refs. [24] and [25].

\subsubsection{Static distortions}

The upper panels in Fig. 10 show the measured static distortions: in four bins of $z$, the average $r \cdot \phi$ residuals (measured minus expected) are plotted as a function of the radius. In order to demonstrate the inadequacy of a fit without the external reference system provided by the RPC overlaps, the $r \cdot \phi$ residuals with respect to such a fit are also shown. The width of the lines in Fig. 10 represents the statistical uncertainty. 

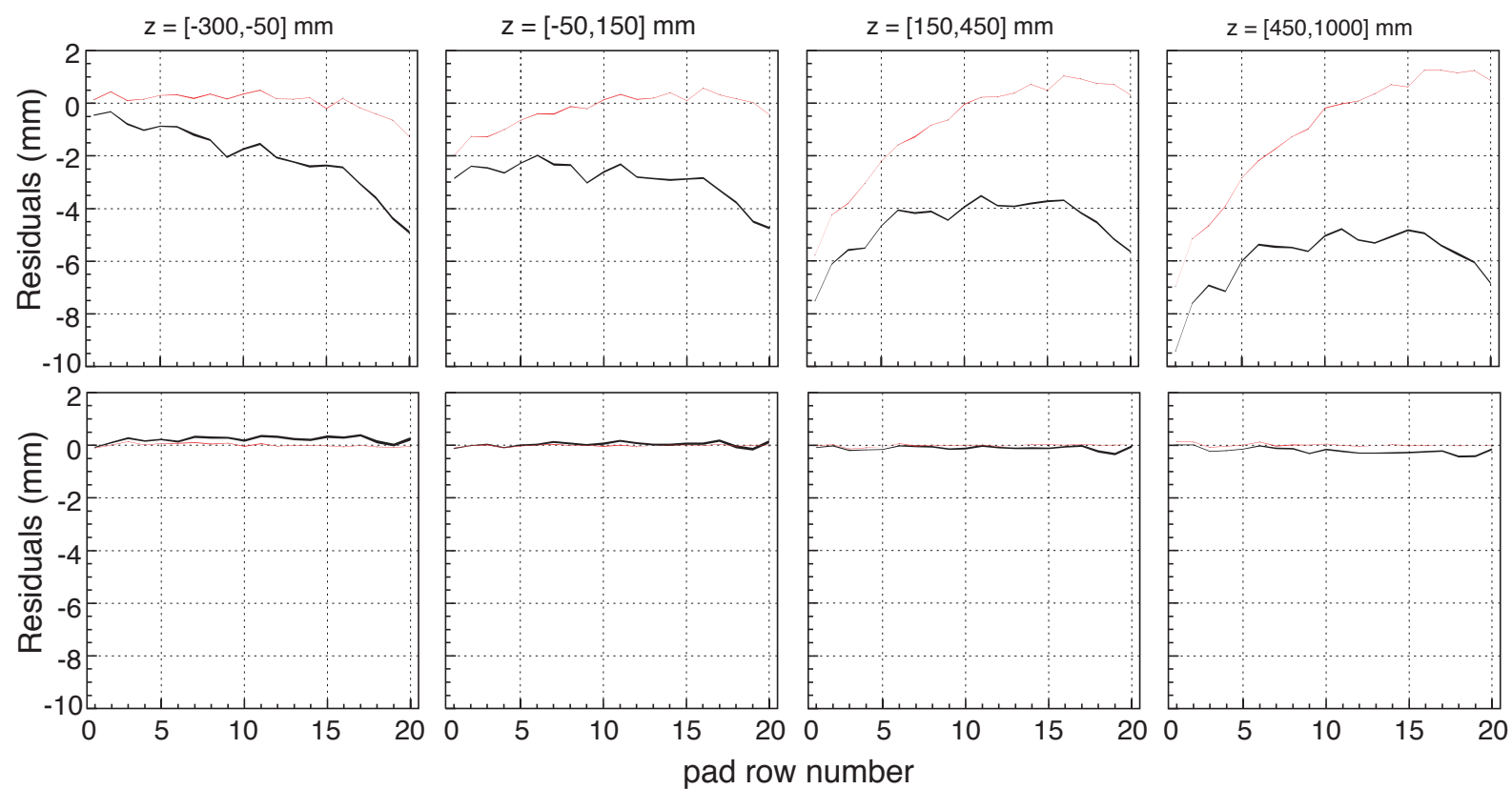

Fig. 10: Average $r \cdot \phi$ residuals [mm] as a function of the radius (pad-row number) for cosmic muons; upper row of panels: before any distortion correction; lower row of panels: after corrections for magnetic field inhomogeneity, high-voltage misalignment, anode-wire 'durchgriff', static 'margaritka' effect, and for the azimuthal shift of the RPC overlaps; the black lines show the $r \cdot \phi$ residuals with respect to the RPC reference system, the grey (red) lines with respect to a fit without the RPC reference system.

\section{Magnetic field inhomogeneity}

The calculation of track distortions from the inhomogeneity of the magnetic field is straightforward and involves no uncertainty.

\section{Homogeneous charge density}

The distortions from the transverse electric field generated by the homogeneous ion charge density from the steady flux of ionizing cosmic muons are negligible. However, we note the good agreement between calculation and observation of distortions in cosmic-muon data-taking right after a ${ }^{83 \mathrm{~m}} \mathrm{Kr}$ calibration: the then much stronger homogeneous charge density caused a distortion which agreed well with expectation, and showed the time dependence expected from the $1.86 \mathrm{~h}$ half-life of the ${ }^{83 \mathrm{~m}} \mathrm{Kr}$ state. Moreover, the study of the distortions after a ${ }^{83 \mathrm{~m}} \mathrm{Kr}$ calibration, and the comparison of their strength with the one that would originate solely from the primary deposition of charges from ${ }^{83 \mathrm{~m}} \mathrm{Kr}$ decay products permitted the measurement of the effective ion amplification factor in the TPC volume as decribed in Ref. [24]. We shall come back to this important point in Section 5.2.2.

\section{High-voltage misalignment}

The electric field distortion that was caused by an accidental misalignment of the high voltage at the membrane of the inner field cage with respect to the high voltage at the corresponding point in the outer field cage, is shown in the $E_{\mathrm{z}}$ and $E_{\mathrm{r}}$ components in Fig. 11. Here, and in all other cases, the electric field was calculated with the POISSON program [23]. Not shown in Fig. 11 is the electric field distortion from two smaller, though significant, misalignments that were caused 

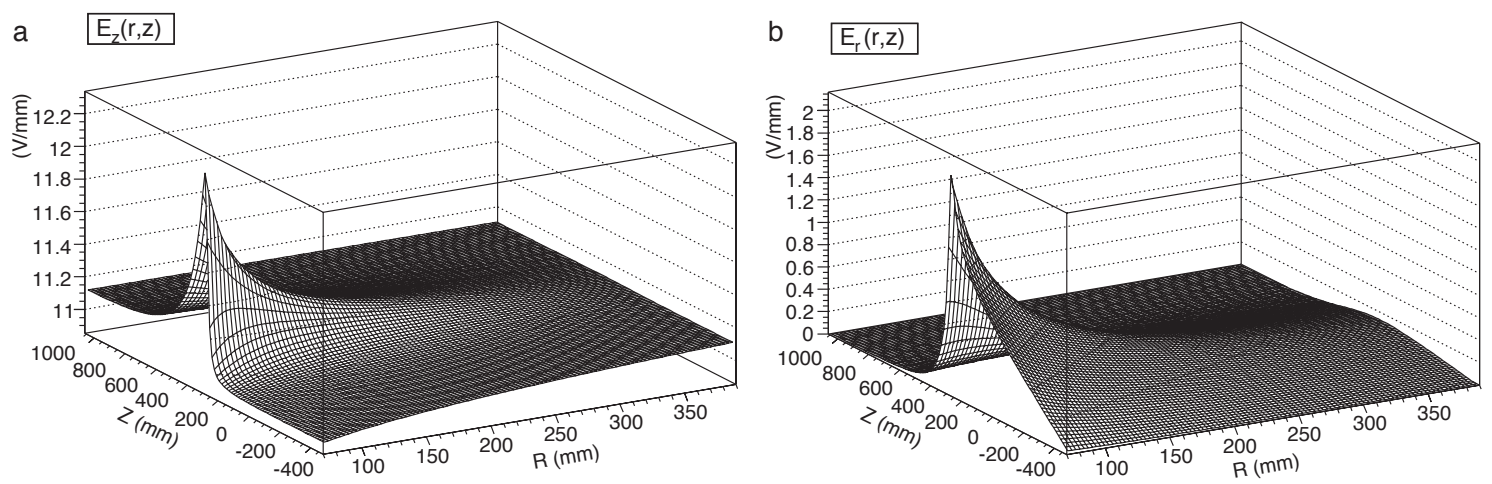

Fig. 11: (a) Longitudinal and (b) radial inhomogeneity of the electrical field from the $167 \mathrm{~V}$ high-voltage misalignment.

by what we came to call the 'field-cage anomalies': a locally reduced resistance in the resistor chain of the inner field cage near the potential strip with $z=-72 \mathrm{~mm}$, and in the resistor chain of the outer field cage near the potential strip with $z=-177 \mathrm{~mm}$. This led at small radius to $\mathrm{a} \sim 1 \%$, and at large radius to a $\sim 0.7 \%$, higher longitudinal field gradient both before and after the discontinuity, and caused an additional transverse electric field which changed sign right at the discontinuity. The amplitude of the field cage anomalies are correlated with the amplitude of the high-voltage misalignment and also with the amplitude of the 'durchgriff' effect that will be discussed next.

The displacements of clusters resulting from the inhomogeneity of the electric field depend strongly on their location inside the TPC volume. They reach several millimetres. The inhomogeneity of the electric field has the following free parameters: the voltage misalignment at the membrane of the inner field cage, and the voltage discontinuities (i.e., deviations from the nominal field-cage potentials) that characterize the field-cage anomalies. From a fit to cosmicmuon distortions, the former was determined to be $167 \mathrm{~V}$, the inner field-cage anomaly to be $-50 \mathrm{~V} /+39 \mathrm{~V}$, and the outer field-cage anomaly to be $-14 \mathrm{~V} /+48 \mathrm{~V}$.

\section{'Durchgriff' effect}

After correction for distortions from the magnetic field inhomogeneity and the high-voltage misalignment, an $r \cdot \phi$ distortion remained with a radial dependence that was largely independent of the $z$ coordinate. It could not be eliminated by changes of the high-voltage misalignment parameters. Its characteristics matched the ones expected from anode-wire 'durchgriff' ${ }^{22}$ ). Because of important consequences for the understanding of dynamic distortions, we take a closer look at the 'durchgriff' effect.

The nominal $-67 \mathrm{~V}$ offset potential of the gating grid was determined from its average distance of $6 \mathrm{~mm}$ from the cathode wires at ground potential, and of $1551 \mathrm{~mm}$ from the HV membrane at $-17302 \mathrm{~V}$. It was calculated on the assumption that the average potential at the location of the cathode wires was zero. This assumption is not valid because the cathode wires do not represent a homogeneous electrode: the wire grid is partially transparent and permits the 'durchgriff' from the positive potential of the anode wires. The situation is depicted for the geometry of the HARP TPC in Fig. 12a. It shows the nominal potential downstream of the

\footnotetext{
${ }^{22)}$ The German noun 'Durchgriff' has no good English counterpart; it is perhaps best translated by 'reach through'.
} 
TPC pads, as a function of the $z$ coordinate. The potential is given for homogenous electrodes (for reference purposes), along a path which is tangential to the surface of the cathode and/or gating-grid wires, and along a path in the middle between two cathode and/or gating-grid wires. The average zero potential is located $3.8 \mathrm{~mm}$ downstream of the cathode wires. The effective potential at the position of the cathode wires is on the average $+96 \mathrm{~V}$. The field cages' potential strips whose middle position coincides with the cathode wire plane, should have been at a potential of $+96 \mathrm{~V}$ and not at ground. The equipotential lines at small and large radii are no longer parallel to the pad plane, and hence produce transverse electric field components which in turn cause static distortions: the 'durchgriff' effect.
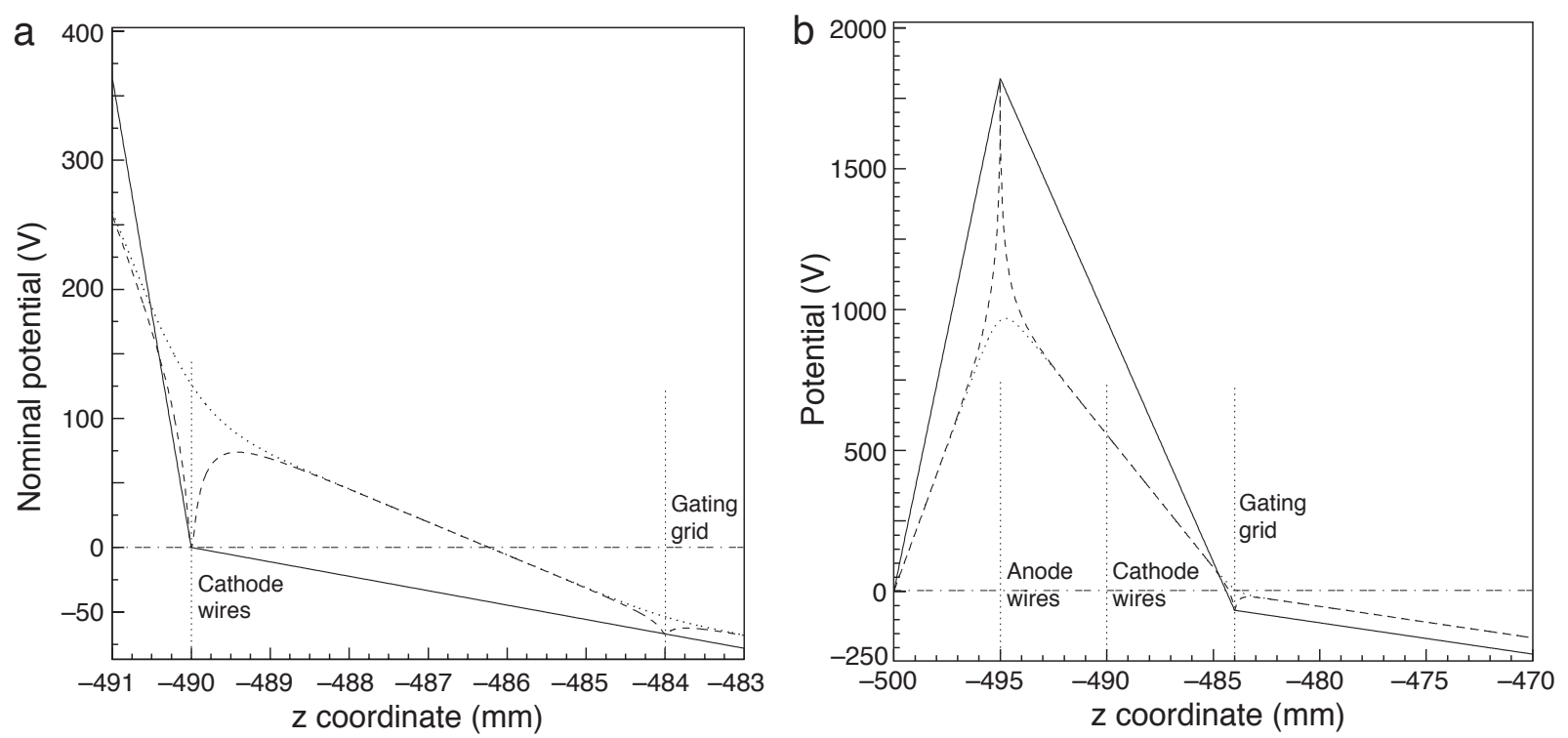

Fig. 12: (a) Potential downstream of the TPC pads with all electrodes at nominal values, as a function of the $z$ coordinate: for homogeneous electrodes (full line), along a path which is tangential to the surface of the cathode and/or gating-grid wires (broken line), and along a path in the middle between two cathode and/or gating-grid wires (dotted line); (b) potential downstream of the TPC pads for floating cathode wires, as a function of the $z$ coordinate.

Since the 'durchgriff' effect depends logarithmically on the diameter of the wires of the electrodes, the standard finite-element method to calculate the potential in the TPC volume is not applicable because the small wire diameters demand unmanageably small element sizes. Instead, we performed first analytic calculations of the 'durchgriff' effect and thus determined the effective potentials of fictitious homogeneous planar electrodes in lieu of the discrete wire grids of the cathode and gating-grid planes. This layout was used in the finite-element calculation which delivered the electric fields in the TPC volume.

The 'durchgriff' effect is localized within some $20 \mathrm{~cm}$ from the pad plane and as such was consistent with the observed $z$-independence of the distortions that remained after correction for distortions from the magnetic field inhomogeneity and the high-voltage misalignment. The observed 'durchgriff' effect, however, was considerably larger than the one calculated for the situation with all electrodes at their nominal values. To explain this finding, we conjecture that the cathode wires were floating during data-taking ${ }^{23)}$. While this notion looks worrying at first

${ }^{23)}$ This conjecture could neither be proven nor disproven by post mortem hardware inspection. 
sight, the functioning of the TPC sense wires is nearly unaffected. To demonstrate the ensuing 'durchgriff' effects, Fig. 12b shows the calculated potential distributions for the case of floating cathode wires. Table 5 lists the pertinent potentials. The 'durchgriff' effect which amounts to $10 \mathrm{~V}$ when all electrodes are connected, is larger by a factor of 5.8 for floating cathode wires.

Table 5: Potentials [V] at the gating-grid location characterizing the 'durchgriff' effect

\begin{tabular}{|l|c|c|}
\hline & Homogeneous electrode & Grid \\
\hline \hline All electrodes connected & -67 & -57 \\
Floating cathode wires & -67 & -9 \\
\hline
\end{tabular}

The best fit of the amplitude of the 'durchgriff' effect, expressed in terms of the effective potential at the position of the gating grid, had a shallow optimum at $-22 \mathrm{~V}$. While the fit excluded the proper connection of all electrodes, the fit with floating cathode wires (i.e., with an effective potential of $-9 \mathrm{~V}$ as stated in Table 5) was nearly as good as the optimum fit of $-22 \mathrm{~V}$. This supports our conjecture that the cathode wires were floating during data taking. We recall that the analysis of the space charge observed after a ${ }^{83 \mathrm{~m}} \mathrm{Kr}$ calibration (see Ref. [24]) confirms independently the notion of floating cathode wires. Furthermore, we note that the analytic calculation uses an ideal configuration, with no experimental uncertainties taken into account on distances, potentials, and wire diameters. We note further that the experimentally determined distortions from the 'durchgriff' effect are the ones after corrections for the inhomogeneity of the magnetic field and the high-voltage misalignment, which also introduce uncertainties. Altogether, we consider $-22 \mathrm{~V}$ compatible with the theoretical prediction of $-9 \mathrm{~V}$.

\section{Static 'Margaritka' effect}

The 'margaritka' effect ${ }^{24}$ ) is caused by a surface charge of positive ions on the central insulator of the TPC wire chambers. It is characterized by a radially outward pointing electric field vector, and is localized to the region close to the TPC wire chamber. The effect has only one parameter: the 'margaritka' potential. The value of the static 'margaritka' potential was determined to be $\sim 350 \mathrm{~V}$.

\section{Performance after static distortion corrections}

The lower panels in Fig. 10 show the final result for the $r \cdot \phi$ residuals of cosmic-muon tracks, after correction of all static distortions and for the azimuthal shift of the RPC overlaps. They are compatible with zero and now, and only now, is there agreement with the $r \cdot \phi$ residuals with respect to a fit without the RPC reference system.

In summary, static distortions up to several millimetres were corrected with a precision of order $150 \mu \mathrm{m}$ throughout the TPC volume.

Figure 13 (a) shows the difference, divided by $\sqrt{2}$, between the independently fitted chargesigned $1 / p_{\mathrm{T}}$ of the half-tracks in the upper and lower hemispheres of through-going cosmic muons, for a full-track $p_{\mathrm{T}}$ range of $1.5<p_{\mathrm{T}}<2.5 \mathrm{GeV} / c$. We note that the upper and lower hemispheres show a small $p_{\mathrm{T}}$ bias with respect to each other. This bias is attributed to remanent

\footnotetext{
${ }^{24)}$ The name stems from the Russian word for a small flower with a bright yellow centre (cf. the ion charge at the wire chamber's central insulator) and white petals arranged in a disc (cf. the radially outward-pointing electric field).
} 
cross-talk, its elimination is dealt with in Section 6.1. The Gaussian resolution is $\sigma\left(1 / p_{\mathrm{T}}\right)=$ $0.46(\mathrm{GeV} / c)^{-1}$ for the fit of cosmic-muon half-tracks without vertex point ${ }^{25)}$. Figure 13 (b) shows the observed linear $p_{\mathrm{T}}$ dependence of the $p_{\mathrm{T}}$ resolution: $\sigma\left(p_{\mathrm{T}}\right) / p_{\mathrm{T}}=0.44 p_{\mathrm{T}}+0.04$.
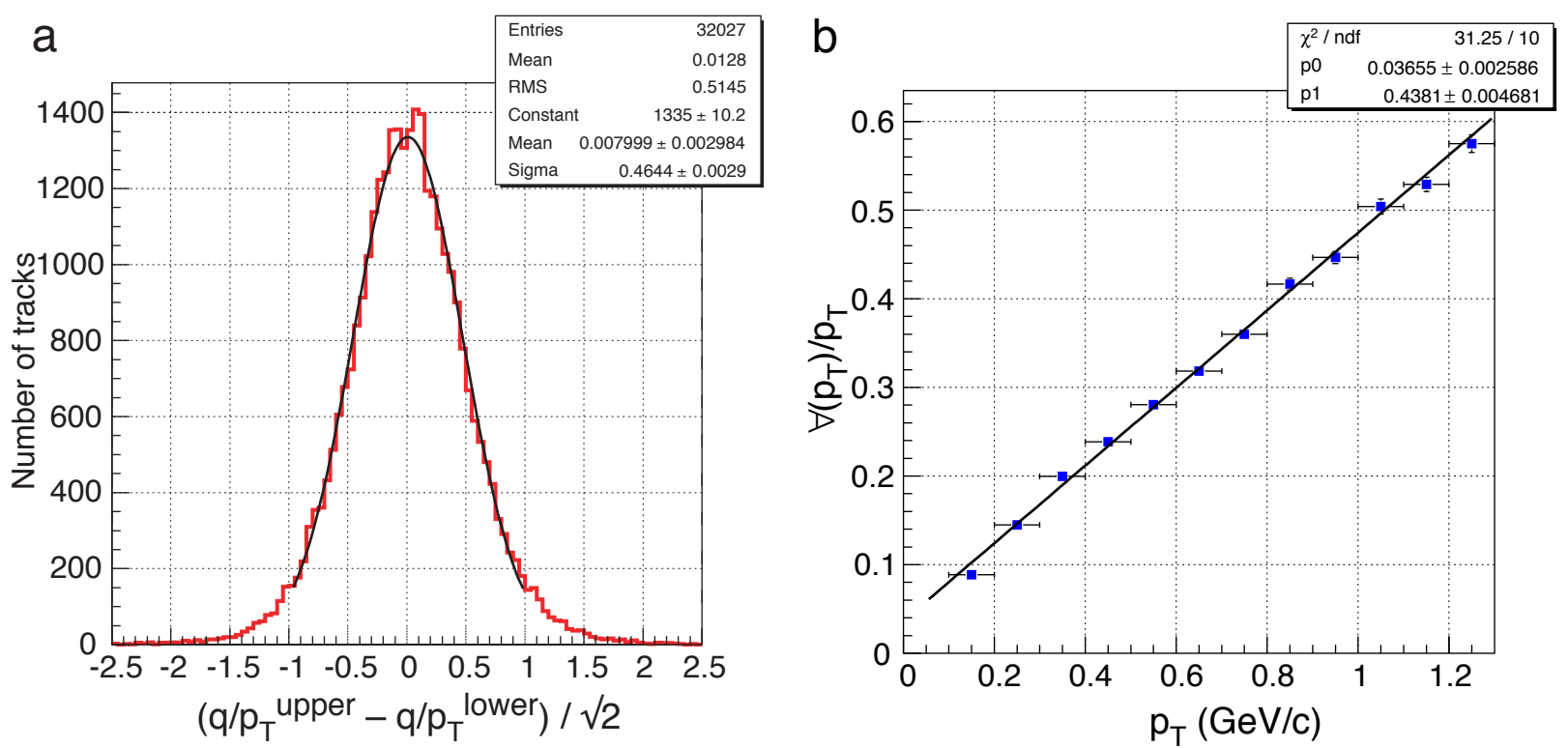

Fig. 13: (a) Difference, divided by $\sqrt{2}$, between the independently fitted charge-signed $1 / p_{\mathrm{T}}$ of the halftracks in the upper and lower hemispheres of through-going cosmic muons; (b) Measured and best-fit $p_{\mathrm{T}}$ dependence of $\sigma\left(p_{\mathrm{T}}\right) / p_{\mathrm{T}}$.

\subsubsection{Dynamic distortions}

We recall that dynamic track distortions, by contrast with static ones, arise solely during the accelerator spill. The experimental evidence for strong dynamic distortions is presented in the upper row of panels of Figs. 14 (positive magnet polarity) and 15 (negative magnet polarity). They show the initial average $r \cdot \phi$ residuals for different pad rows from small to large radius as a function of the event time during the accelerator spill. However, as pointed out in the introductory part of this section, the initial $r \cdot \phi$ residuals are only a first approximation because the track curvature that was used for their measurement, is biased. The middle row of panels in Figs. 14 and 15 show the $r \cdot \phi$ distortions determined with the correct track curvature that is obtained after the convergence of an iterative procedure of distortion correcting and track re-fitting. We note the strong time dependence of the distortions; that the distortions are in the $10 \mathrm{~mm}$ domain; that the amplitude of the distortions depends on the data set; that the distortions at large radius have opposite sign to the distortions at small radius; and that the distortions change sign with the polarity of the magnetic field. We note further that the lines which represent the residuals with respect to a fit without the RPC reference system, are unsuitable for assessing dynamic distortions. The width of the lines represents the statistical uncertainty.

We identified and modelled three different sources of dynamic distortions.

${ }^{25)}$ This resolution is a priori not representative for physics tracks since no dynamic distortions are involved. 

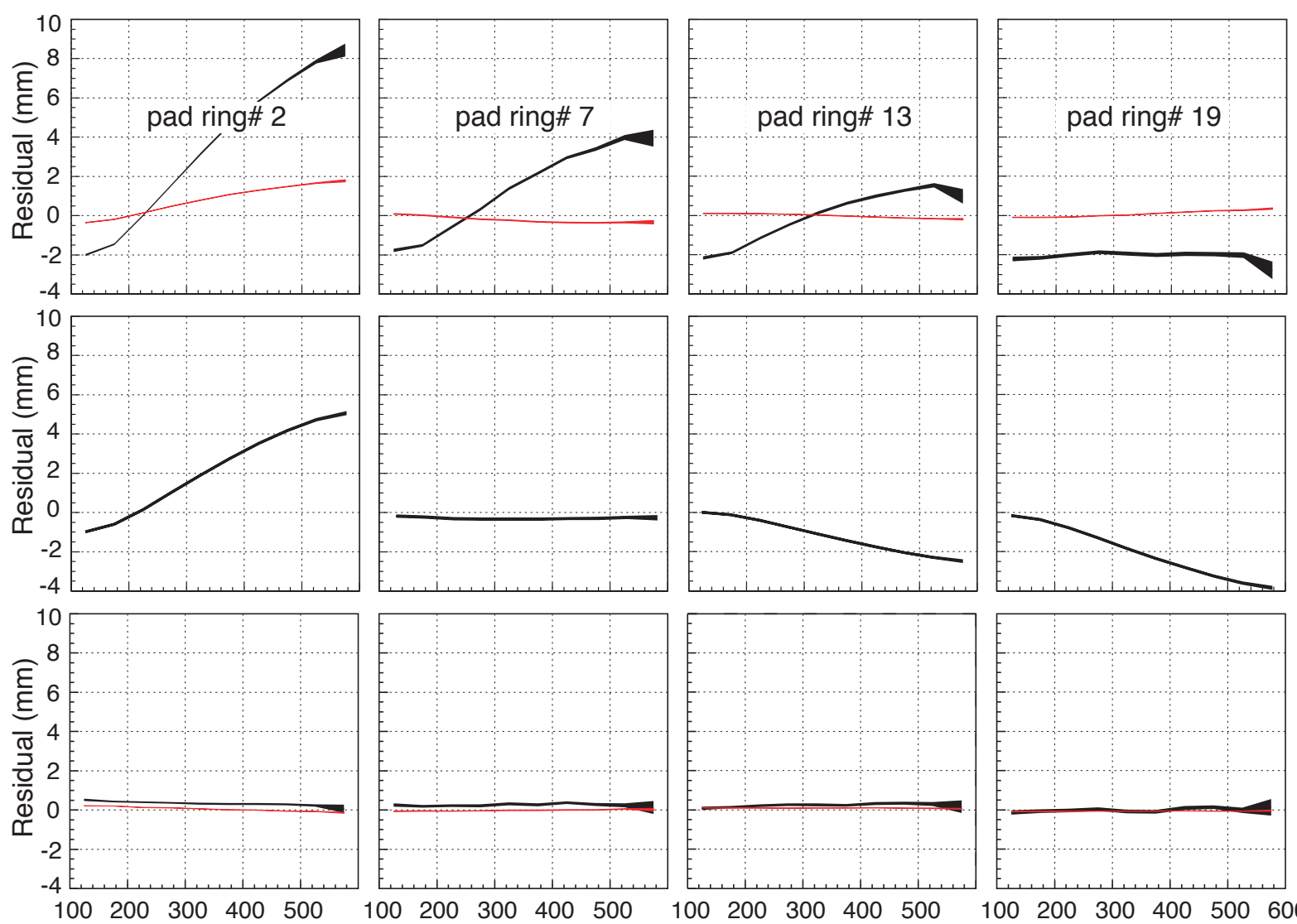

Event time in spill (ms)

Fig. 14: 'Thin $\mathrm{Be}+8.9 \mathrm{GeV} / c$ ' data; upper row of panels: initial average $r \cdot \phi$ residuals [mm], after static distortion corrections, for different pad rows from small to large radius, as a function of the event time [ms] in the accelerator spill; middle row of panels: same after the convergence of the iterative procedure (see text); lower row of panels: same after correction of dynamic distortions and for the azimuthal shift of the RPC overlaps; the black lines show the $r \cdot \phi$ residuals with respect to the RPC reference system, the grey (red) lines with respect to a fit without the RPC reference system. 

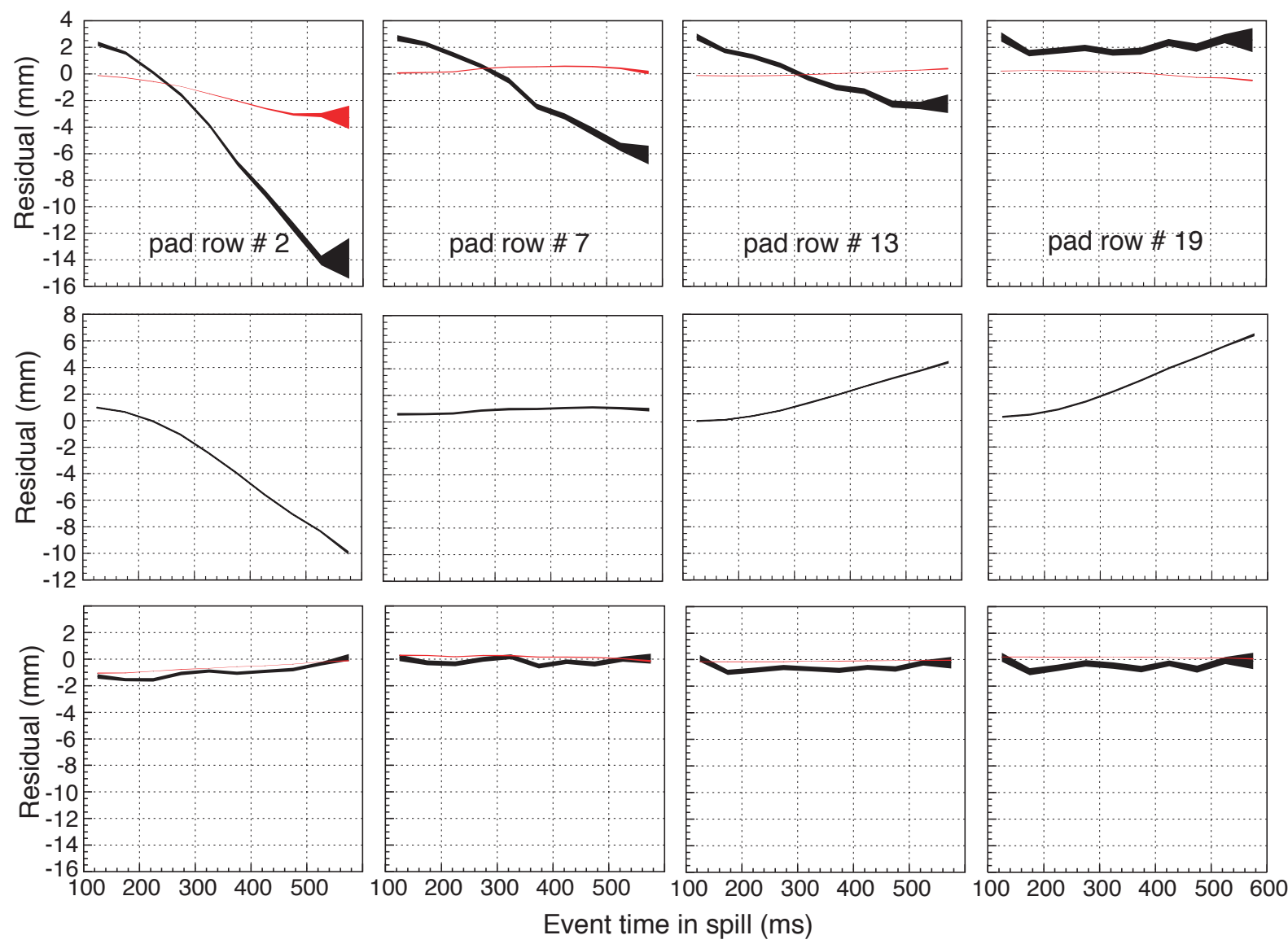

Fig. 15: 'Thin $\mathrm{Be}-8.0 \mathrm{GeV} / c$ ' data; upper row of panels: initial average $r \cdot \phi$ residuals [mm], after static distortion corrections, for different pad rows from small to large radius, as a function of the event time [ms] in the accelerator spill; middle row of panels: same after the convergence of the iterative procedure (see text); lower row of panels: same after correction of dynamic distortions and for the azimuthal shift of the RPC overlaps; the black lines show the $r \cdot \phi$ residuals with respect to the RPC reference system, the grey (red) lines with respect to a fit without the RPC reference system. 


\section{Line charge}

The TPC's inner field cage does not extend throughout the TPC volume, it ends at $z=286 \mathrm{~mm}$. Therefore, all beam particles which did not interact in the target, will ionize the TPC gas downstream of the inner field cage and generate a line charge of $\mathrm{Ar}^{+}$ions. These ions move toward the HV membrane which is located at $z=1067 \mathrm{~mm}$ at the downstream end of the TPC. The time-dependent line-charge density gives rise to a transverse electric field, and thus to dynamic track distortions. For normal running conditions, though, the dynamic distortions arising from this line-charge density can be neglected.

\section{Dynamic 'Margaritka' effect}

From the conjecture that dynamic distortions are caused by the build-up of $\mathrm{Ar}^{+}$ions in the TPC volume, one expects physics tracks at the start of the spill to be free from dynamic distortions. Therefore, for such tracks one expects to see no further distortion after correction for static distortions.

We draw attention to the feature in the upper panels in Figs. 14 and 15 whereby at the beginning of the spill the distortions do not start at zero (note that all static corrections have been applied), but at an offset.

This offset arises from the dynamic 'margaritka' effect: during the spill, the surface charge of positive ions on the central insulator of the TPC wire chambers is larger than outside the spill. The charge density reaches rapidly equilibrium and is in the modelling considered constant throughout the spill. The size of the effect is determined by the dynamic 'margaritka' potential which is data-set dependent. For the 'thin $\mathrm{Be}+8.9 \mathrm{GeV} / c$ ' data set, the 'margaritka' potential is $\sim 500 \mathrm{~V}$ on top of the static 'margaritka' potential of $\sim 350 \mathrm{~V}$, with a variation of $\sim 20 \%$ between TPC sectors.

\section{'Stalactite' effect}

By far the largest dynamic distortion was caused by the build-up of $\mathrm{Ar}^{+}$ions in the active TPC volume, because of a malfunctioning of the gating grid.

The gating grid had been intended for two purposes: (i) to prevent in its closed state electrons from entering from the TPC volume into the amplification region; ideally, the electron transparency should have been zero in the gating grid's closed state, and $100 \%$ in its open state; and (ii) to prevent in its closed state ions from drifting from the amplification region back into the TPC volume; ideally, the ion transparency should have been zero in the gating grid's closed state.

In the gating grid's open state, all wires were at the offset potential of $-67 \mathrm{~V}$ which should have coincided with the potential of the drift field at the gating grid's $z$ position (but was not the case as explained in the discussion of the 'durchgriff' effect). In the gating grid's closed state, a voltage swing of $\pm 35 \mathrm{~V}$ on top of the offset potential was applied between the two wire planes of the gating grid. A simulation of the functioning of the gating grid gave the following results [26]: (i) while the gating grid was reasonably closed for electrons for zero magnetic field, it was transparent at the $80 \%$ level for a magnetic field of $0.7 \mathrm{~T}$; and (ii) while the gating grid should have been closed for ions, it was transparent at the $1 \%$ level.

With $N_{\mathrm{e}}$ the average number of electrons created per second and cubic centimetre in the TPC volume, the charge flux at the sense wire plane is ${ }^{26)} N_{\mathrm{e}} Q_{\mathrm{e}} T_{\mathrm{e}} L \mathrm{C} \mathrm{cm}^{-2} \mathrm{~s}^{-1}$, where $Q_{\mathrm{e}}=$ $1.6 \times 10^{-19} \mathrm{C}$ is the electron charge, $T_{\mathrm{e}} \sim 0.8$ is the gating grid's electron transparency in

\footnotetext{
${ }^{26)}$ Assuming, in first approximation, a uniform creation of electrons along $z$.
} 
the 'closed' state, and $L=156.2 \mathrm{~cm}$ is the distance between the sense-wire plane and the HV membrane. This charge flux is amplified by $A \sim 2 \times 10^{4}$, and a fraction $T_{\text {ion }} \sim 0.01$ returns to the TPC volume where it drifts with a velocity $v_{\text {ion }} \sim 150 \mathrm{~cm} / \mathrm{s}$ toward the HV membrane, creating an $\mathrm{Ar}^{+}$charge density of $N_{\mathrm{e}} Q_{\mathrm{e}} T_{\mathrm{e}} L A T_{\mathrm{ion}} / v_{\text {ion }}$ : the initial charge density $N_{\mathrm{e}} Q_{\mathrm{e}}$ is amplified by a factor of $\sim 170$.

Unfortunately, the actual characteristics of the gating grid were even worse than described so far. With cathode wires floating rather than connected to ground, the gating-grid wires take the function of the cathode wires and the ion transparency increases from about 1\% to 3-4\% [26] Hence the $\mathrm{Ar}^{+}$charge density is by a factor of $\sim 600$ larger than the initial electron charge density.

This large ion amplification factor agrees well with the one inferred from the ion charge density that causes static distortions of cosmic-ray muons after a ${ }^{83 \mathrm{~m}} \mathrm{Kr}$ calibration, as discussed in Section 5.2.1.

We have the phenomenon of an ion column which grows out of the sense-wire plane and drifts slowly toward the HV membrane, continuously fed from its base: the 'stalactite' effect ${ }^{27}$. With a drift velocity of $150 \mathrm{~cm} / \mathrm{s}$, the maximum ion drift distance is $60 \mathrm{~cm}$ during the $400 \mathrm{~ms}$ long accelerator spill. After the end of the spill, the stalactite eventually dissipates in the HV membrane. At the beginning of the next spill, a new stalactite starts growing.

It was this stalactite growth in the TPC drift volume which was the physical cause of the observed dynamic distortions. If the beam intensity is constant during the accelerator spill, dynamic distortions from stalactite growth increase approximately linearly with time.

For a given setting of the data-taking, i.e., for a given beam momentum, beam polarity and target, the average spatial distribution of secondaries, including beam muons, is fixed. The radial charge-density profile of the stalactite is given by the transverse projection of the density of amplified primary electrons of whatever origin. Accordingly, for the modelling of the stalactite, its radial profile is determined by adding up the numbers of ADC counts of all observed clusters within the TPC volume ${ }^{28)}$, with no regard to their association with a reconstructed track.

Our algorithm has rotational symmetry but allows for different ion densities in different TPC sectors.

An important parameter is the beam intensity as a function of the time within the spill. The relevant information is available from two beam scalers which measure (i) the relative time of the event with respect to the start of the spill, and (ii) the number of incoming beam particles recorded since the previous event trigger. For a sufficiently precise modelling of the dynamic distortions, the times with 'gating grid open' and 'gating grid closed' must be taken into account (more ions are produced during 'gating grid open'). This is done by using the instantaneous rate of incoming beam particles 'BS' and the instantaneous trigger rate 'NEV' as parameters.

We found the following parametrization of the stalactite's charge density as a function of radius $R$ and time $t$ effective:

$$
\begin{aligned}
Q(R, t)= & 5 \cdot 10^{-14} \times Q_{0}(R) \\
& \times(1+\exp (-t / \tau)) \\
& \times(0.004 \cdot \mathrm{BS} \cdot(1-\beta)+0.1 \cdot \mathrm{NEV} \cdot \beta) \\
& \times C_{\text {sect }},
\end{aligned}
$$

\footnotetext{
${ }^{27)}$ The name stems from the close analogy to a stalactite: a column of calcium salt which grows, fed from its base, from the roof of a cave.

${ }^{28)}$ Bad pads are skipped but their contributions are taken into account by normalizing to the number of good pads in the respective pad row.
} 
where the first line gives an overall normalization and the radial dependence, the second line describes a time dependence that was found advantageous to be included, the third line gives the dependence on the beam intensity and event rate, and the fourth line gives the sector-dependent normalization. Typical numerical values for 'thin $\mathrm{Be}+8.9 \mathrm{GeV} / c$ ' data are $\beta=0.41, \tau=$ $0.28 \mathrm{~s}$, and $C_{\text {sect }}=0.26$ (sectors differed by typically $10 \%$ ). The drift velocity of the positive ions was a further free parameter. A broad optimum was obtained with $v_{\text {ion }} \simeq 100 \mathrm{~cm} / \mathrm{s}$, independent of the data set.

Detailed studies led to improvements beyond Eq. (9). The stalactite's growth takes into account its broadening in the transverse electric field caused primarily by the high-voltage misalignment. It was also found advantageous to alter slightly the radial charge distribution by a factor $\left(1+\alpha r_{\min } / r\right)$, where $r_{\min }=73 \mathrm{~mm}$ is the minimal radius of the pad plane's sensitive region. Best results were obtained with $\alpha \sim-0.65^{29)}$.

The parameters of the stalactite must be separately optimized for each data set.

Technically, the longitudinal charge profile of the stalactite is approximated by means of $25 \mathrm{~mm}$ long contiguous 'discs', which emanate in discrete steps from the sense wire plane and move downstream, one right behind the other. The linear superposition of the fields of the discs constitutes the electric field generated by the stalactite.

The electric field distortion that was caused by the 'stalactite' at the end of the spill, is shown in the $E_{\mathrm{z}}$ and $E_{\mathrm{r}}$ components in Fig. 16 for the specific case of the 'thin $\mathrm{Be}+8.9 \mathrm{GeV} / c$ ' data taking. This data taking was characterized by quite some decrease of beam intensity toward the end of the spill which is reflected in a lower field distortion at the upstream end of the TPC (i.e., at large negative $z$ ). We note the inhomogeneity in the longitudinal field component that causes changes of the electron drift velocity, and the inhomogeneity in the radial field component that causes dynamic track distortions.
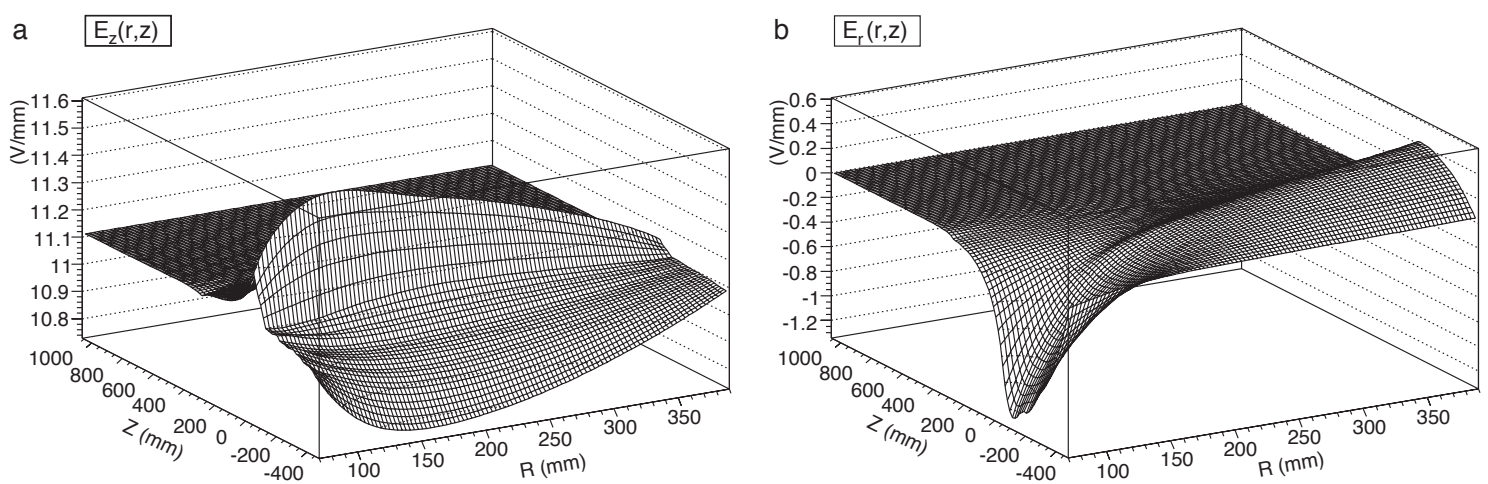

Fig. 16: 'Thin $\mathrm{Be}+8.9 \mathrm{GeV} / c^{\prime}$ ' data; (a) longitudinal and (b) radial inhomogeneity of the electrical field from the 'stalactite' at the end of the spill.

\footnotetext{
${ }^{29)}$ We conjecture as likely origin the stalactite's initial division into separate sectors, caused by the dead region around the 'spokes' of the TPC wire chamber, and later partial coalescence of the segments, together with the increase of the stalactite's charge density with time at small radius; the larger ion velocity, primarily at small radius, at the stalactite's front and the smaller ion velocity at its end, in conjunction with the actually employed constant ion velocity, is conjectured to cause the time-dependence in Eq. (9) [27].
} 


\section{Performance after all distortion corrections}

The average $r \cdot \phi$ residuals, after correction of all dynamic distortions and for the azimuthal shift of the RPC overlaps, are shown in the lower panels of Figs. 14 and 15.

The quality of the overall correction of static and dynamic track distortions is perhaps best demonstrated by Fig. 17. It shows that the average $1 / p_{\mathrm{T}}$ is flat across the spill, and the chargesigned $1 / p_{\mathrm{T}}$ spectrum is the same at the start and at the end of the spill. In order to achieve such congruence, the entire TPC volume must be systematically under control to better than $300 \mu \mathrm{m}$.
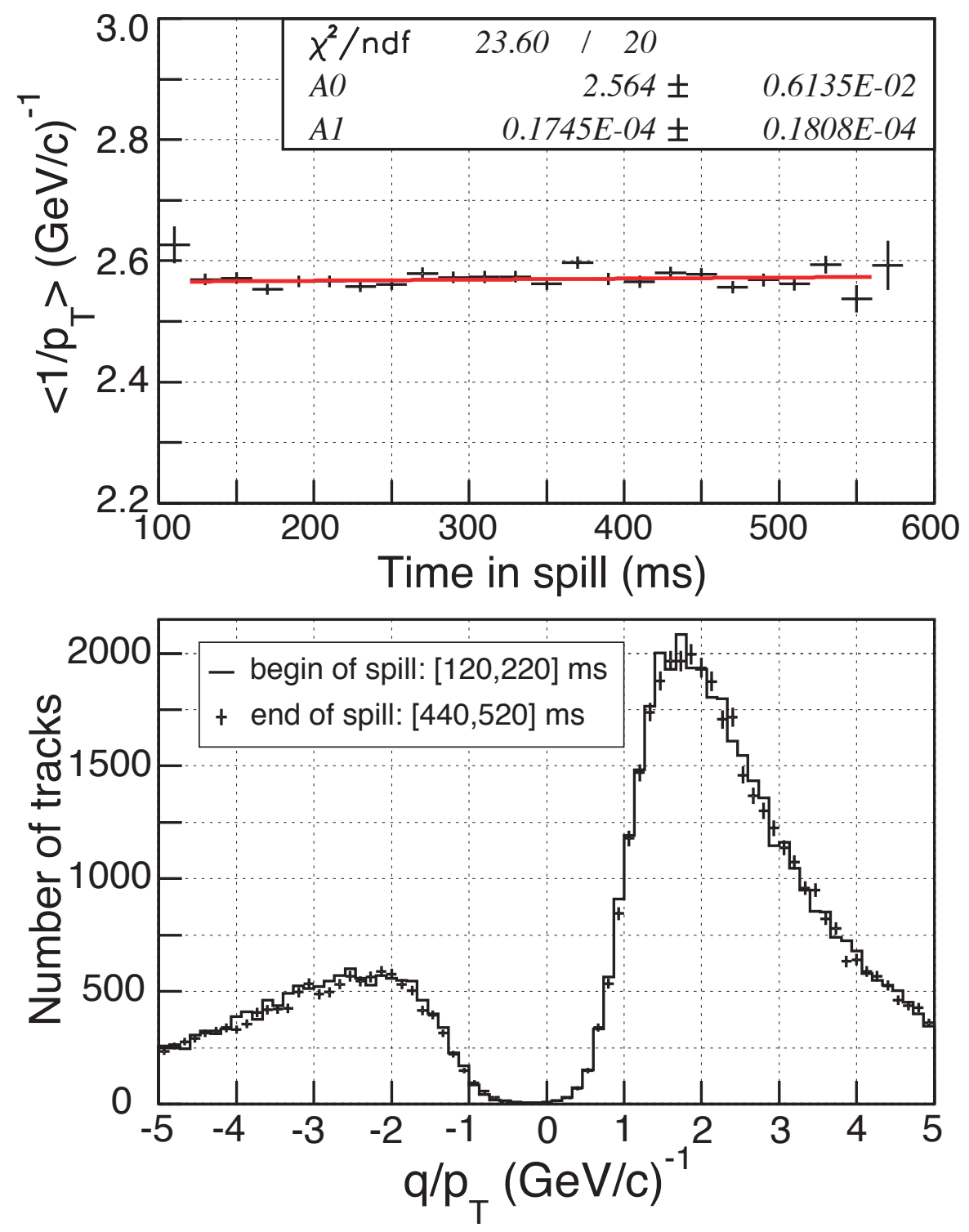

Fig. 17: 'Thin $\mathrm{Be}+8.9 \mathrm{GeV} / c$ ' data; upper panel: average $1 / p_{\mathrm{T}}$ of tracks with reconstructed positive charge, as a function of the event time in the spill; lower panel: charge-signed $1 / p_{\mathrm{T}}$ spectra at the start and at the end of the spill. 


\subsubsection{Synopsis of distortions from field inhomogeneities}

Table 6 presents a concise summary of static and dynamic TPC distortions in the HARP TPC, in both $r$ and $r \cdot \phi$. The distortions depend on $r$ and $z$, and in part on time, and range from zero to the indicative values given in the table. We call attention to the signs of the corrections as a function of radius, and their dependence on the magnetic field polarity.

Table 6: Indicative values of static and dynamic distortions in millimetres, in $r$ and $r \cdot \phi$; in case of a double sign, the upper sign refers to positive beam polarity and the lower sign to negative beam polarity.

\begin{tabular}{|l|c|c|c|c|}
\hline & \multicolumn{2}{|c|}{ Small radius } & \multicolumn{2}{|c|}{ Large radius } \\
\hline & $\Delta r$ & $\Delta(r \cdot \phi)$ & $\Delta r$ & $\Delta(r \cdot \phi)$ \\
\hline \hline Magnetic field inhomogeneity & +0.6 & $\mp 0.2$ & +3 & $\mp 1$ \\
Homog. charge density of $1 \times 10^{-16} \mathrm{C}^{-\mathrm{cm}^{3}}$ & +0.01 & \pm 0.03 & -0.01 & $\mp 0.03$ \\
High-voltage misalignment & -3 & $\mp 9$ & -1 & $\mp 3$ \\
'Durchgriff' effect & +0.5 & \pm 1.5 & -0.5 & $\mp 1.5$ \\
'Margaritka' effect & -0.7 & $\mp 2$ & -0.009 & $\mp 0.03$ \\
Line charge & -0.02 & $\mp 0.07$ & -0.003 & $\mp 0.009$ \\
'Stalactite' effect & +3 & \pm 9 & -1 & $\mp 3$ \\
\hline
\end{tabular}

Of the static track distortions, only the one caused by the homogeneous charge density from the steady flux of cosmic muons is negligible. Of the dynamic track distortions, only the linecharge effect is negligible.

\section{SUMMARY OF THE PHYSICS PERFORMANCE}

The TPC's central task is the momentum reconstruction. Determinant requirements are a correct momentum scale and an adequate momentum resolution.

The good understanding of the detector performance is complemented by its Monte Carlo simulation with the GEANT4 program [28]. This simulation comprises beam particles and the secondaries from their interactions in the targets, and permits the comparison and consistent assessment of observed and expected detector performance.

In the simulation, the experimental situation is imposed: the $r \cdot \phi$ position of TPC clusters is smeared according to the observed distributions, clusters are eliminated according to the observed losses, the incoming beam is reproduced as observed, the same fit algorithms are applied for data and simulated data.

The performance results reported in this section are based on events taken across the entire accelerator spill.

\subsection{Momentum scale}

The momentum determined from the $p_{\mathrm{T}}$ and $\theta$ of reconstructed TPC tracks can be cross-checked against the momentum determined from $\mathrm{d} E / \mathrm{d} x$, and the momentum determined from the time of flight measured in the RPCs.

After the corrections for static and dynamic distortions, the consequences of only partial correction for cross-talk still remain and may lead to a bias in momentum scale. Since locally uncorrected distortions of cluster positions know neither the $p_{\mathrm{T}}$ nor the charge sign of tracks, a bias of momentum scale is naturally expressed as a sagitta bias. Accordingly, the correct 
momentum scale was enforced by means of a small correction of the absolute value of the sagitta which was applied with opposite sign for positively and negatively charged particles. This sagitta correction which amounted to a shift in $1 / p_{\mathrm{T}}$ of $\sim 0.05(\mathrm{GeV} / c)^{-1}$ (depending on the TPC sector and on the polar angle $\theta$ ), was determined with high precision from the requirement that $\pi^{+}$and $\pi^{-}$with the same time of flight have the same momentum. Within this model, the momentum scale depends only on the magnetic field strength that we estimate to be known to better than $1 \%$. Including a possible inadequacy of the sagitta distortion model, we consider that the momentum scale is correct to better than $2 \%$, both for positively and negatively charged particles. The momentum determination from $\mathrm{d} E / \mathrm{d} x$ is in agreement with this conclusion.

\section{2 $p_{\mathrm{T}}$ resolution}

Figure 18 (a) shows the difference of the inverse transverse momentum of positive particles with $0.6<\beta<0.75$ and $45^{\circ}<\theta<65^{\circ}$ from the measurement in the TPC and from the determination from RPC time of flight with the proton-mass hypothesis. The background from pions and kaons is very small: this is suggested by the analogous plot for negative particles that were selected with the same cuts (genuine antiprotons are absent among negative particles). Subtracting quadratically from the convoluted resolution of $0.27(\mathrm{GeV} / c)^{-1}$ the contribution from the time-of-flight resolution of the RPC, gives a net TPC resolution of $\sigma\left(1 / p_{\mathrm{T}}\right)=0.20(\mathrm{GeV} / c)^{-1}$.

Figure 18 (b) shows the net TPC resolution $\sigma\left(1 / p_{\mathrm{T}}\right)$ resolution as a function of of their relative velocity $\beta$, Fig. 18 (c) the same as a function of the polar angle $\theta$. The agreement with the expectation from a Monte Carlo simulation is satisfactory. The resolution $\sigma\left(1 / p_{\mathrm{T}}\right)$ is typically $20 \%$ and worsens towards small $\beta$ and small polar angle $\theta$. The reason for this is that in both cases the position error of the beam point increases because of increased multiple scattering in materials before the protons enter the TPC.

\section{3 $\mathbf{d} E / \mathbf{d} x$ versus momentum}

We show in Fig. 19 the $\mathrm{d} E / \mathrm{d} x$ of positive secondaries. Corrections for gas pressure and temperature changes are applied. The agreement with the theoretical expectation is good and consistent with a momentum scale that is correct within $2 \%$.

\subsection{Time of flight versus momentum}

Figure 20 shows the velocity, as determined by time of flight from the RPCs, against the momentum measured in the TPC. The data shown in panels (a) and (b) extend over the full acceptance of the barrel RPCs and follow nearly perfectly the theoretical expectation ${ }^{30)}$. The data in panel (c) show tracks in the RPC padring 7 on a logarithmic scale which is intended to make visible small admixtures of kaons and deuterons.

\subsection{Pion-electron separation}

The electron-pion separation capability of the TPC and RPC tandem is demonstrated in Fig. 21 where the velocity of tracks with momenta between 95 and $215 \mathrm{MeV} / \mathrm{c}$ is plotted against the energy loss of the tracks as measured in the TPC. Electron and pion clusters are well separated in velocity over the full momentum range in which they cannot be distinguished by $\mathrm{d} E / \mathrm{d} x$ in the TPC.

${ }^{30)} \mathrm{A}$ small offset of the proton band is well understood and is not related to the momentum scale [6]. 

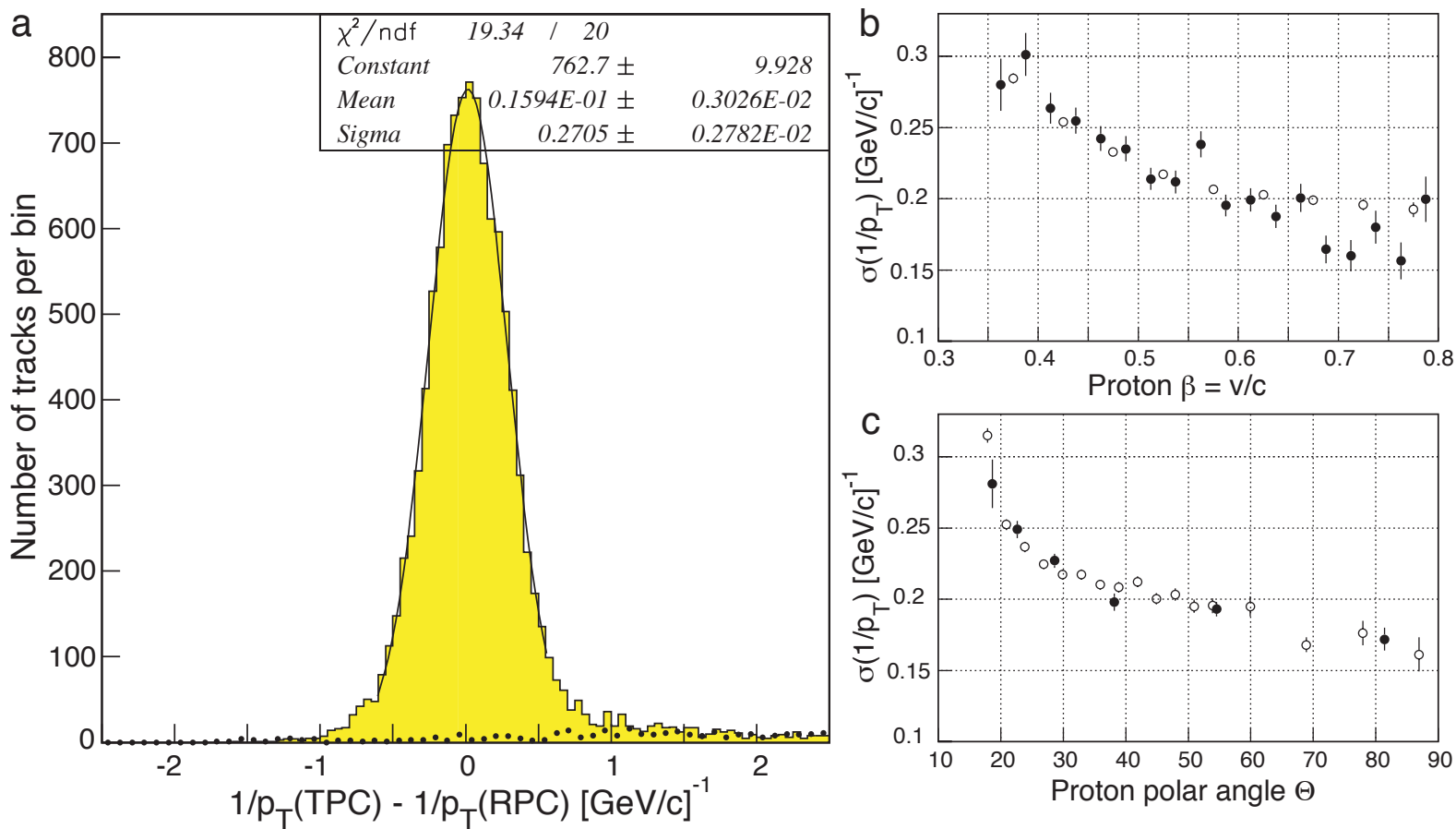

Fig. 18: (a) Difference of the inverse transverse momenta of positive (shaded histogram) and negative (black points) particles from the measurement in the TPC and from the determination from RPC time of flight, for $0.6<\beta<0.75$ and for $45^{\circ}<\theta<65^{\circ}$; the positive particles are protons, with a very small background from pions and kaons; (b) $\sigma\left(1 / p_{\mathrm{T}}\right)$ of protons with $45^{\circ}<\theta<65^{\circ}$ as a function of their relative velocity $\beta$; (c) $\sigma\left(1 / p_{\mathrm{T}}\right)$ of protons with $0.6<\beta<0.75$ as a function of their polar angle $\theta$; black points denote data, open circles Monte Carlo simulation.

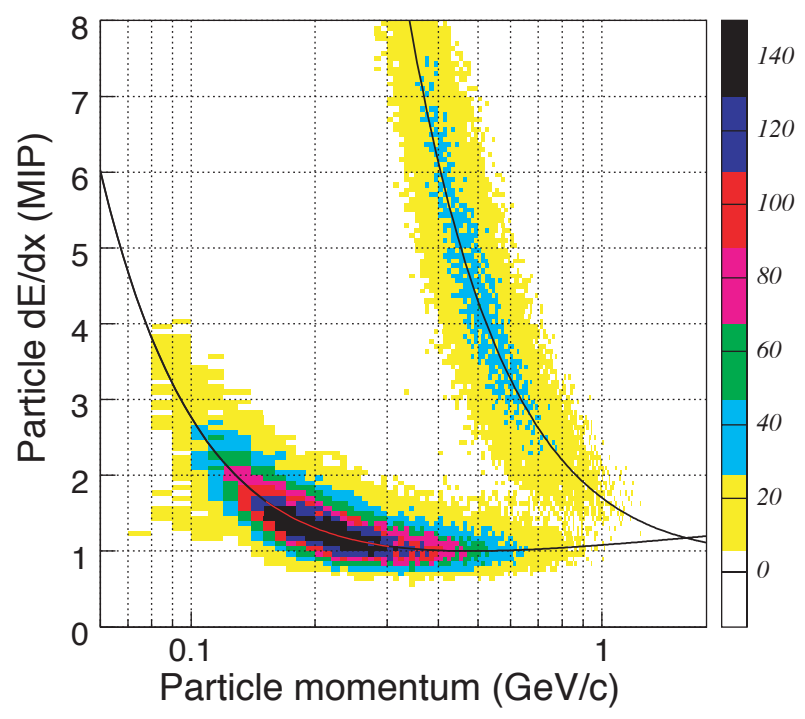

Fig. 19: Specific ionization $\mathrm{d} E / \mathrm{d} x$ [in units of minimum-ionizing pulse height] versus momentum $[\mathrm{GeV} / c]$ for positive secondaries, together with the theoretical expectations for pions and protons. 

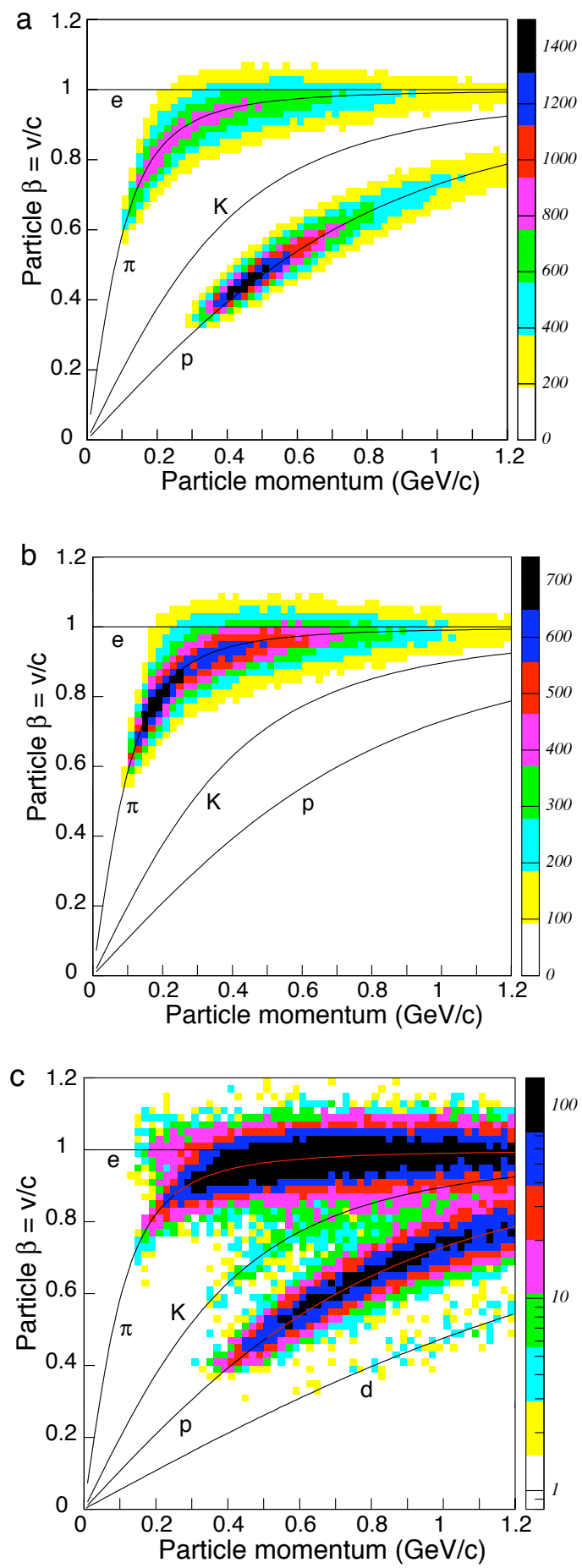

Fig. 20: Velocity versus particle momentum for positive (a) and negative (b) tracks; for positive tracks, panel (c) shows tracks in the RPC padring 7 on a logarithmic scale which makes visible small admixtures of kaons and deuterons; the lines show the theoretical relations between particle velocity and momentum for electrons, pions, kaons, protons, and deuterons. 

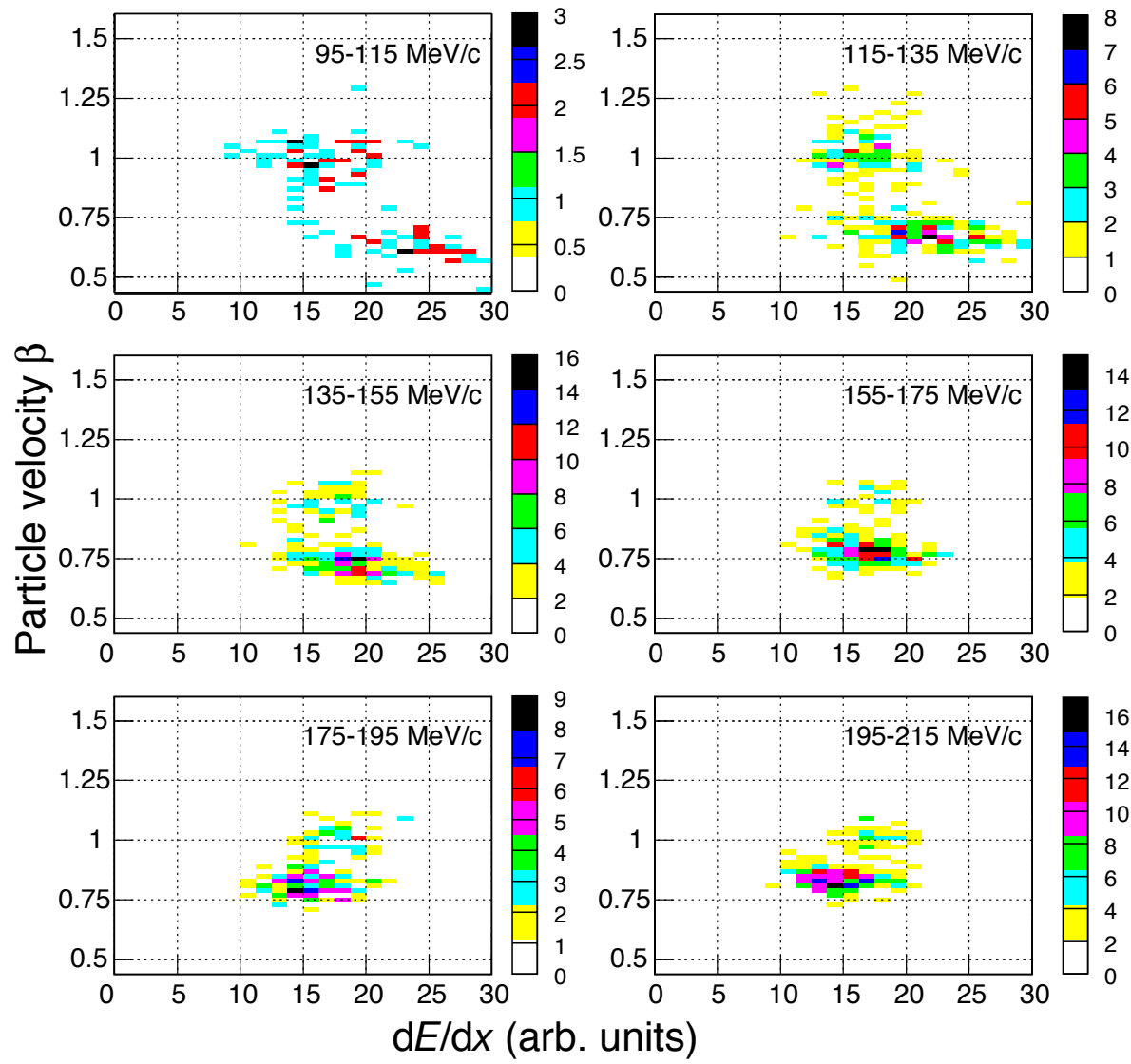

Fig. 21: Normalized velocity of positive secondary particles as measured by the RPCs, for particle momenta in the range 95-215 MeV/c, versus their specific ionization $\mathrm{d} E / \mathrm{d} x$ measured in the TPC. 


\section{SUMMARY}

The physics performance of the HARP TPC is described, with emphasis on a number of corrections that had to be applied to the data to ascertain optimal results. It is shown that large static and dynamic track distortions can be corrected to insignificance. The only remanent problem was partially irreducible electronics cross-talk between pads which led to an $r \cdot \phi$ resolution that was worse than planned, yet in no way impairing correct and precise differential hadron production cross-sections. In particular, a momentum resolution of better than $20 \%$ was achieved for secondary protons of $1 \mathrm{GeV} / c$ momentum, emitted perpendicularly to the beam direction.

\section{ACKNOWLEDGements}

We are indebted to P. Zucchelli for his contributions, and dedication, to the design and construction of the HARP TPC; to J.-C. Legrand for his contributions to the design of the TPC readout electronics; to M. Delattre, C. Detraz, J.L. Loquet, J. Mulon, and M. Scandurra for their help with the mechanical design and construction of the TPC; to R. Veenhof for simulation work that helped to understand the operational characteristics of the TPC; to V. Serdiouk for his contributions to the construction and operation of the TPC; and to M. Chizhov and Ch. Wiebusch for their contributions in the early stages of TPC calibration work. Last, but not least, we thank S. Leech O'Neale for her professional proof-reading of this paper.

\section{REFERENCES}

[1] M.G. Catanesi, et al., Nucl. Instr. and Meth. A 571 (2007) 527.

[2] The HARP Collaboration, Nucl. Instr. and Meth. A 571 (2007) 564.

[3] V. Ammosov, et al., HARP Memo 06-101, http://cern.ch/dydak/WhiteBook.pdf

[4] V. Ammosov, et al., Nucl. Instr. and Meth. A 571 (2007) 562.

[5] S.R. Amendolia, et al., Nucl. Instr. and Meth. A 252 (1986) 392.

[6] V. Ammosov, et al., Nucl. Instr. and Meth. A 578 (2007) 119

[7] W. Blum and L. Rolandi, Particle Detection with Drift Chambers, Springer Verlag, Berlin and Heidelberg, 1993.

[8] D. Decamp, et al., Nucl. Instr. and Meth. A 294 (1990) 121.

[9] A. De Min, et al., IEEE Trans. Nucl. Sci. 42 (1995) 491.

[10] S. Afanasiev, et al., Nucl. Instr. and Meth. A 430 (1999) 210.

[11] K. Fujii, et al., Nucl. Instr. and Meth. A 264 (1988) 297.

[12] N.I. Chernov and G.A. Ososkov, Comput. Phys. Commun. 33 (1984) 329.

[13] F. Dydak and Yu. Nefedov, HARP Memo 04-002, http://cern.ch/dydak/GeneralizedLSF.ps

[14] K. Fujii, et al., Track reconstruction with the TRISTAN-TOPAZ TPC, KEK preprint 8724; JLC Physics Group, Introduction to helical track manipulations (Internal Report, 6 June 1997)

[15] V. Ammosov, et al., HARP Memo 06-103, http://cern.ch/dydak/GLSFsequel.pdf

[16] D. Buskulic, et al., Nucl. Instr. and Meth. A 360 (1995) 481.

[17] S.R. Amendolia, et al., Nucl. Instr. and Meth. 217 (1983) 317.

[18] W. Blum, et al., Nucl. Instr. and Meth. A 252 (1986) 407.

[19] S.R. Amendolia, et al., Nucl. Instr. and Meth. A 244 (1986) 516.

[20] S.R. Amendolia, et al., Nucl. Instr. and Meth. A 283 (1989) 573.

[21] F. Dydak, HARP memo 03-001, http://cern.ch/dydak/TPCdistortions.ps 
[22] F. Dydak, A. Krasnoperov and Yu. Nefedov, HARP Memo 03-002, http://cern.ch/dydak/TPCdistortions2.ps

[23] J.H. Bilen, et al., Program POISSON/Superfish, Report LA-UR-96-1834 (1996; revised 2004).

[24] I. Boyko, et al., HARP Memo 05-101, http://cern.ch/dydak/TPCdistortions3.pdf

[25] V. Ammosov, et al., HARP Memo 06-107, http://cern.ch/dydak/TPCdistortions4.pdf

[26] R. Veenhof, http://cern.ch/rjd/Harp/gateset.html, and private communication.

[27] We thank L. Rolandi who suggested a change of ion drift velocity inside the stalactite.

[28] S. Agostinelli, et al., Nucl. Instr. and Meth. A 506 (2003) 250. 